DIPLOMARBEIT

\title{
A new spectrometer for grazing incidence X-ray fluorescence for the characterization of Arsenic implants and $\mathrm{Hf}$ based high- $\kappa$ layers
}

\author{
ausgeführt am \\ Atominstitut \\ der Technischen Universität Wien \\ Stadionallee 2 \\ 1020 Wien
}

unter der Anleitung von

Ao.Univ.Prof. Dipl.-Ing. Dr.techn. Christina Streli

durch

Dieter Ingerle

Floridsdorfer Hauptstraße 22/4/9

1210 Wien

Wien, 23. Oktober 2015 


\section{Kurzfassung}

Röntgenfluoreszenzanalyse unter streifendem Einfall (GIXRF) benutzt den Effekt der totalen externen Reflektion von Röntgenstrahlen an einer glatten, polierten Oberfläche. Aufgrund der geringen Eindringtiefe von nur wenigen Nanometern bei kleinem Einfallswinkel kann GIXRF die Zusammensetzung der Probe nahe der Oberfläche untersuchen. Weiters kann die Methode Informationen über die totale Dosis und Tiefenverteilung der Elemente liefern, indem die Intensität der winkelabhängige Röntgenfluoreszenz bei verschiedenen Winkeln gemessen wird. Da die gewonnene Information über die Tiefenverteilung nicht eindeutig ist, müssen in den Auswertevorgang, der darin besteht gemessene und simulierte Kurven zu vergleichen, weitere Daten von einer anderen Methode einfliessen.

In der vorliegenden Arbeit wird eine im Rahmen der von der EU geförderten European Integrated Activity of Excellence and Networking for Nano and MicroElectronics Analysis (ANNA) entwickelte, gebaute und getestete Messkammer für GIXRF präsentiert. Weiters wurde eine Mess- und Steuersoftware sowie eine Messvorschrift entwickelt und getestet.

Um die Funktion der entwickelten Kammer zu testen, wurden Messungen an Ultra Shallow Junctions (USJ) durchgeführt. Als ergänzende Methode für die Ermittlung des Tiefenprofils wurde Sekundärionen-Massenspektrometrie (SIMS) eingesetzt. Die Messungen der totalen implantierten Arsen-Dosis wurde mit Ergebnissen von Instrumenteller Neutronenaktivierungsanalyse (INAA) und SIMS verglichen. Weiters wurden Schichten von Hafniumverbindungen auf Silizium untersucht und die Resultate mit den Ergebnissen von anderen Methoden, durchgeführt durch die ANNA Partner, verglichen.

Teile dieser Arbeit wurden in den folgenden Publikationen veröffentlicht:

1. D. Ingerle, F. Meirer, N. Zoeger, G. Pepponi, D. Giubertoni, G. Steinhauser, P. Wobrauschek, C. Streli, A new spectrometer for grazing incidence Xray fluorescence for the characterization of Arsenic implants and Hf based high-k layers, Spectrochimica Acta Part B: Atomic Spectroscopy 65 (6) (2010) 429-433. doi:10.1016/j.sab.2004.04.014

2. G. Pepponi, D. Giubertoni, M. Bersani, F. Meirer, D. Ingerle, G. Steinhauser, C. Streli, P. Hoenicke, B. Beckhoff, Grazing incidence x-ray fluorescence and secondary ion mass spectrometry combined approach for the characterization of ultrashallow arsenic distribution in silicon, Journal of Vacuum Science \& Technology B: Microelectronics and Nanometer Structures 28 (1) 
(2010) C1C59. doi:10.1116/1.3292647

3. D. Giubertoni, G. Pepponi, B. Beckhoff, P. Hoenicke, F. Gennaro, F. Meirer, D. Ingerle, G. Steinhauser, M. Fried, P. Petrik, A. Parisini, M. A. Reading, C. Streli, J. van den Berg, M. Bersani, Multi-technique characterization of arsenic ultra shallow junctions in silicon within the ANNA consortium, AIP Conference Proceedings 1173 (2009) (2009) 45-49. doi:10.1063/1.3251258 


\section{Abstract}

Grazing Incidence X-ray Fluorescence Analysis (GIXRF) takes advantage of the total external reflection of X-rays on a smooth, polished surface. Due to the penetration depth of only a few nanometers at very small incidence angles, GIXRF is able to probe the elemental composition in the near surface region of the sample. Furthermore by adjusting the angle of incidence and measuring the angledependent X-ray fluorescence signals, the technique is able to provide information on the total dose and depth distribution of the elements. The depth profile information is ambigous, thus the evaluation process, which consists of fitting the measured data to simulations, needs additional input from another technique.

In the present work a GIXRF measuring chamber is presented, which was designed, constructed and tested within the context of the EC funded European Integrated Activity of Excellence and Networking for Nano and Micro-Electronics Analysis (ANNA) . Moreover an acquisition and control software and a measurement protocol were developed and tested.

GIXRF measurements for the characterization of Ultra Shallow Junctions (USJ) were used to ascertain the performance of the new instrument. Secondary Ion Mass Spectrometry (SIMS) was used as a complementary technique for the depth profile evaluation. The dose quantification of the implanted Arsenic was compared with Instrumental Neutron Activation Analysis (INAA) and SIMS. Furthermore Hafnium based high $\mathrm{k}$ layers on Silicon were analyzed and the results compared to other techniques of the ANNA partners.

Part of this work has been published in the following publications:

1. D. Ingerle, F. Meirer, N. Zoeger, G. Pepponi, D. Giubertoni, G. Steinhauser, P. Wobrauschek, C. Streli, A new spectrometer for grazing incidence $X-$ ray fluorescence for the characterization of Arsenic implants and Hf based high-k layers, Spectrochimica Acta Part B: Atomic Spectroscopy 65 (6) (2010) 429-433. doi:10.1016/j.sab.2004.04.014

2. G. Pepponi, D. Giubertoni, M. Bersani, F. Meirer, D. Ingerle, G. Steinhauser, C. Streli, P. Hoenicke, B. Beckhoff, Grazing incidence x-ray fluorescence and secondary ion mass spectrometry combined approach for the characterization of ultrashallow arsenic distribution in silicon, Journal of Vacuum Science \& Technology B: Microelectronics and Nanometer Structures 28 (1) (2010) C1C59. doi:10.1116/1.3292647

3. D. Giubertoni, G. Pepponi, B. Beckhoff, P. Hoenicke, F. Gennaro, F. Meirer, 
D. Ingerle, G. Steinhauser, M. Fried, P. Petrik, A. Parisini, M. A. Reading, C. Streli, J. van den Berg, M. Bersani, Multi-technique characterization of arsenic ultra shallow junctions in silicon within the ANNA consortium, AIP Conference Proceedings 1173 (2009) (2009) 45-49. doi:10.1063/1.3251258 


\section{Acknowledgements}

Firstly, I would like to express my sincere gratitude to my advisor and group leader Dr. Christina Streli for encouragement and support, but also patience.

I am thankful to Dr. Peter Wobrauschek, who is still, despite being officially "im Ruhestand" (in retirement), a very enthusiastic and active member of the group, always ready to share his experience.

I want also to mention and express my gratitude to former members of the X-ray group, who worked with me and helped on the project, namely Dr. Norbert Zöger and Dr. Florian Meirer, as well as Dr. Giancarlo Pepponi, who often joined our discussions, when we were left wondering what is actually happening during the measurements.

I thank Dr. Peter Kregsamer for playing the role of advocatus diaboli and the ensuing fruitful discussions.

Special thanks also to all other members of the X-ray group for support and assistance in time of need.

I owe my gratitude to my family, i.e. wife and parents, for their continued support and patience.

Finally, I acknowledge the support of this work by the European Commission Research Infrastructure Action under the Sixth Framework Programme (FP6) through the Integrated Infrastructure Initiative ANNA (project no. 26134). 


\section{Contents}

\begin{tabular}{lll}
\hline Introduction & 10
\end{tabular}

2 Fundamentals 12

$2.1 \quad$ Nature and Production of X-rays . . . . . . . . . . . . . . . 12

2.1 .1 Continuous radiation . . . . . . . . . . . . . 12

2.1 .2 Characteristic radiation . . . . . . . . . . . 14

2.2 Interaction of X-rays with Matter . . . . . . . . . . . . . . . . . . . . . . . . . . . .

2.2.1 Photoelectric Absorption . . . . . . . . . . . . . 15

2.2 .2 Inelastic / Compton Scattering . . . . . . . . . . . . . . 18

$2.2 .3 \quad$ Elastic / Rayleigh Scattering . . . . . . . . . . . . . . . . . . 18

$2.3 \quad$ Principles of X-ray Fluorescence Analysis . . . . . . . . . . . . . . 18

$2.3 .1 \quad$ X-ray sources . . . . . . . . . . . . . . . . . 19

$2.3 .2 \quad$ Energy dispersive X-ray detectors . . . . . . . . . . . . . 20

2.4 Principles of Grazing Incidence X-ray Fluorescence . . . . . . . 22

2.4 .1 Total Reflection X-ray Fluorescence . . . . . . . . . . . . . . 22

2.4 .2 Monochromatization of X-rays . . . . . . . . . . . . . . . . 24

2.4 .3 Grazing Incidence X-ray Fluorescence . . . . . . . . . . . . 25

\begin{tabular}{lll}
\hline & Instrumentation & 30
\end{tabular}

3.1 X-ray source . . . . . . . . . . . . . . . . . . . . . . 30

3.2 Vacuum chamber and sample stage . . . . . . . . . . . . . . . . . . . . . . . . . . . . . . . . .

3.3 XRF detector . . . . . . . . . . . . . . . . . . . . 34

4 Control Software 35

4.1 Devices . . . . . . . . . . . . . . . . . . . . . 35

4.1 .1 Motors . . . . . . . . . . . . . . . . . 35

4.1 .2 Detectors . . . . . . . . . . . . . . . . . . 36

4.2 Configuration file . . . . . . . . . . . . . . . . 36

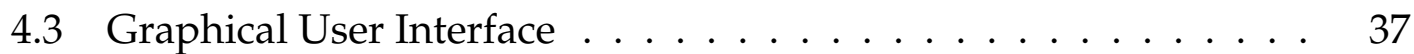

4.3 .1 Main Window . . . . . . . . . . . . . . . . . . 37

4.3 .2 Scan Window . . . . . . . . . . . . . . . . . 38

$\begin{array}{lll}5 & \text { Results and discussion } & 40\end{array}$

5.1 Measuring protocol . . . . . . . . . . . . . . . 42

$5.1 .1 \quad$ Procedures I: System startup . . . . . . . . . . . . . . . 42

5.1 .2 Procedures II: Measurement . . . . . . . . . . . . . . 43 
5.2 Arsenic Implants $\ldots \ldots \ldots \ldots \ldots \ldots \ldots$

5.3 Hafnium Oxide Layers $\ldots \ldots \ldots \ldots \ldots \ldots$

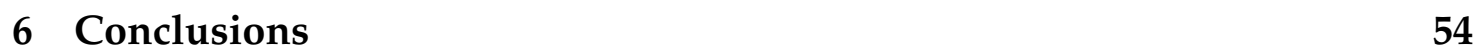

\begin{tabular}{ll}
\hline References & 55
\end{tabular}

\begin{tabular}{ll}
\hline A Software Reference & $\mathbf{6 0}$
\end{tabular}

A.1 Builtin Commands . . . . . . . . . . . . . . . . . . . 60

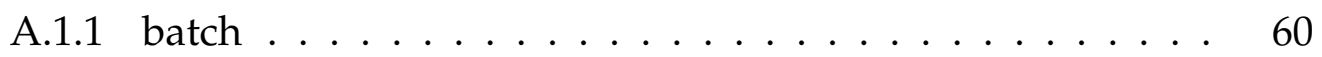

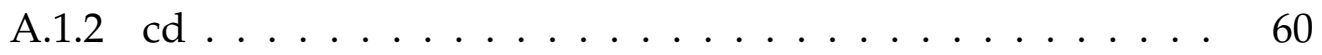

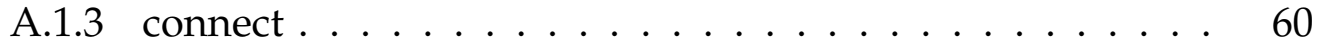

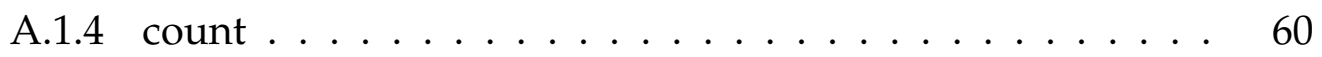

A.1.5 disconnect . . . . . . . . . . . . . . . . . . 60

A.1.6 $\mathrm{mca} \ldots \ldots \ldots \ldots \ldots$

A.1.7 motor. . . . . . . . . . . . . . . . . . . . 60

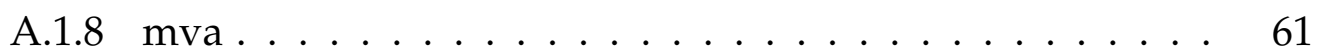

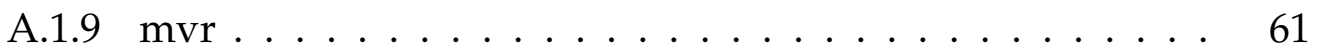

A.1.10 pwd . . . . . . . . . . . . . . . . 61

A.1.11 save. . . . . . . . . . . . . . . . . . . . . 61

A.1.12 scan . . . . . . . . . . . . . . . . . . . 61

A.1.13 scan2d . . . . . . . . . . . . . . . . . . . . 61

A.1.14 sleep . . . . . . . . . . . . . . . . . . . . 62

A.1.15 status . . . . . . . . . . . . . . . . . . . . 62

A.2 Device Commands . . . . . . . . . . . . . . . . . . . 62

A.2.1 connect. . . . . . . . . . . . . . . . . . . . . 62

A.2.2 disconnect . . . . . . . . . . . . . . . . . . . . 62

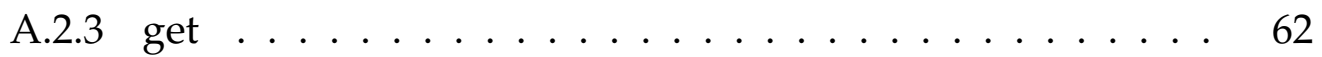

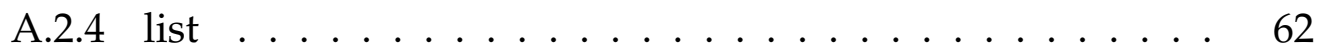

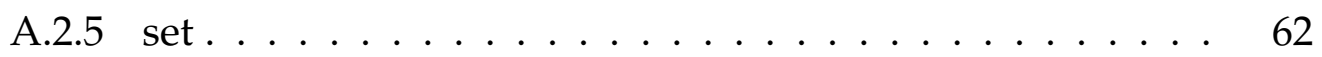

A.2.6 status . . . . . . . . . . . . . . . . . . . . . . . 62

A.3 Motors . . . . . . . . . . . . . . . . . . . . . 63

A.3.1 mva . . . . . . . . . . . . . . . . . . 63

A.3.2 mvr . . . . . . . . . . . . . . . . 63

A.4 Detectors . . . . . . . . . . . . . . . . . . . . . . 63

A.4.1 count . . . . . . . . . . . . . . . . . 63

A.4.2 roiadd . . . . . . . . . . . . . . . . . . 63

A.4.3 roidel . . . . . . . . . . . . . . . . . . . 63 
A.4.4 save. . . . . . . . . . . . . . . . . . . . . 63

A.5 Configuration file $\ldots \ldots \ldots \ldots \ldots \ldots \ldots \ldots \ldots$ 


\section{Introduction}

In the early 1990s de Boer (partly with van den Hoogenhof as co-author) published a seminal series of articles on Total reflection X-ray fluorescence (TXRF) and Glacing-incidence X-ray fluorescence (GIXRF) of thin-layered samples. In these papers a detailed description and analysis of X-ray standing wave effects and the angle dependent measurement curves is given [1, 2, 3]. Also a detailed formalism for the calculation of the emitted fluorescene intensities was introduced [4]. During the following years they continued this series (partly with Leenaers) and presented the idea of a combined GIXRF and X-ray reflectometry (XRR) analysis and an instrument for this combined Glancing incidence X-ray analysis (GIXA) [5, 6, 7, 8]. But layer thicknesses and device sizes used in semiconductor device fabrication at this time were probably still too large, i.e. in the range of some hundred nanometers, to fully utilise the potential of this technique. Furthermore the calculation of emitted fluorescence intensities is quite complicated and thus was probably too time-consuming, for the computers at this time, to employ it in routine analysis.

About ten years later GIXRF was "rediscovered" by Pepponi et al. [9] as a complementary technique for the analysis of ultra-shallow junctions. The measurements presented in this paper were carried out at a synchrotron beamline, as there was no suitable measurement chamber for the laboratory. Nowadays implantation depths and layer thicknesses for semiconductor device fabrication reach the range of some nanometers and thus seem ideally suited for GIXRF, which has its largest sensitivity on and a few nanometer below the surface. Also in order to support the shrinking of device sizes, new materials beside Silicon Oxide are developed, tested and introduced for gate oxides in field-effect transistors, which poses additional challenges for the currently employed techniques. Thus the idea was born to develop a suitable GIXRF instrument and to compare the performance of the technique with other methods within the European Integrated Activity of Excellence and Networking for Nano and Micro- Electronics Analysis (ANNA).

The instrument developed and presented in the present work consists of two parts. Firstly, a vacuum chamber with X-ray source, monochromator, motors and XRF detector suitable for the specific needs of GIXRF, namely angle steps of $0.005^{\circ}$ and smaller in the range of $0^{\circ}$ to $2^{\circ}$ and X-ray count rates from almost zero to ten thousand counts per second and more. And secondly an acquisition and control software, which automates the tedious task of rotating the sample 
in hundreds of steps and acquire a XRF spectrum at each step in the course of several hours.

Part of this work has been published in [10], [11] and [12]. It was supported by the European Commission Research Infrastructure Action under the FP6 "Structuring the European Research Area" Programme through the Integrated Infrastructure Initiative ANNA (project no. 26134). 


\section{Fundamentals}

\subsection{Nature and Production of X-rays}

The term X-rays describes electromagnetic radiation, which typically has an energy in the range $100 \mathrm{eV}$ to $100 \mathrm{keV}$ or equivalently a wavelength ranging from 0.01 to 10 nanometers, as energy $E$, frequency $v$ and wavelength $\lambda$ have the simple relation:

$$
E=h v=\frac{h c}{\lambda}
$$

where $h$ is the Planck constant $\left(\sim 4.136 \times 10^{-15} \mathrm{eVs}\right)$ and $c$ is the speed of light. $X$-rays at the high energy end of the spectrum are called hard X-rays. Part of this energy range is overlapping with $\gamma$-rays, thus it is also common practice to distinguish these two types of radiation by their source, i.e. X-rays are emitted by electrons, while $\gamma$-rays are emitted by the atomic nucleus. In a similar manner Xrays of lower energy are called soft $\mathrm{X}$-rays, and the energy range is overlapping with the range of ultraviolet (UV) radiation.

The spectrum of a typical X-ray tube as shown in Figure 1 shows two distinct features, a continuous radiation "background" and sharp characteristic lines. This characteristic is due to different production processes, which will be described in the following two sections.

\subsubsection{Continuous radiation}

Whenever a free charged particle is accelerated, it emits electromagnetic radiation. This radiation is called cyclotron radiation, synchrotron radiation or bremsstrahlung (i.e. "braking radiation"), depending on the speed of the particle and the type of acceleration.

Bremsstrahlung is typically caused by the deceleration of an electron in the anode material of an X-ray tube. The electron is deflected by the electric field of an atomic nucleus and loses kinetic energy (Figure 2). This energy difference is equal to the energy $E_{v}$ of the emitted photon, i.e.

$$
E_{v}=E_{i}-E_{f}
$$

where $E_{i}$ and $E_{f}$ are the energies of the electron before and after the interaction.

As there are no quantization conditions for this process, the radiation is pro- 


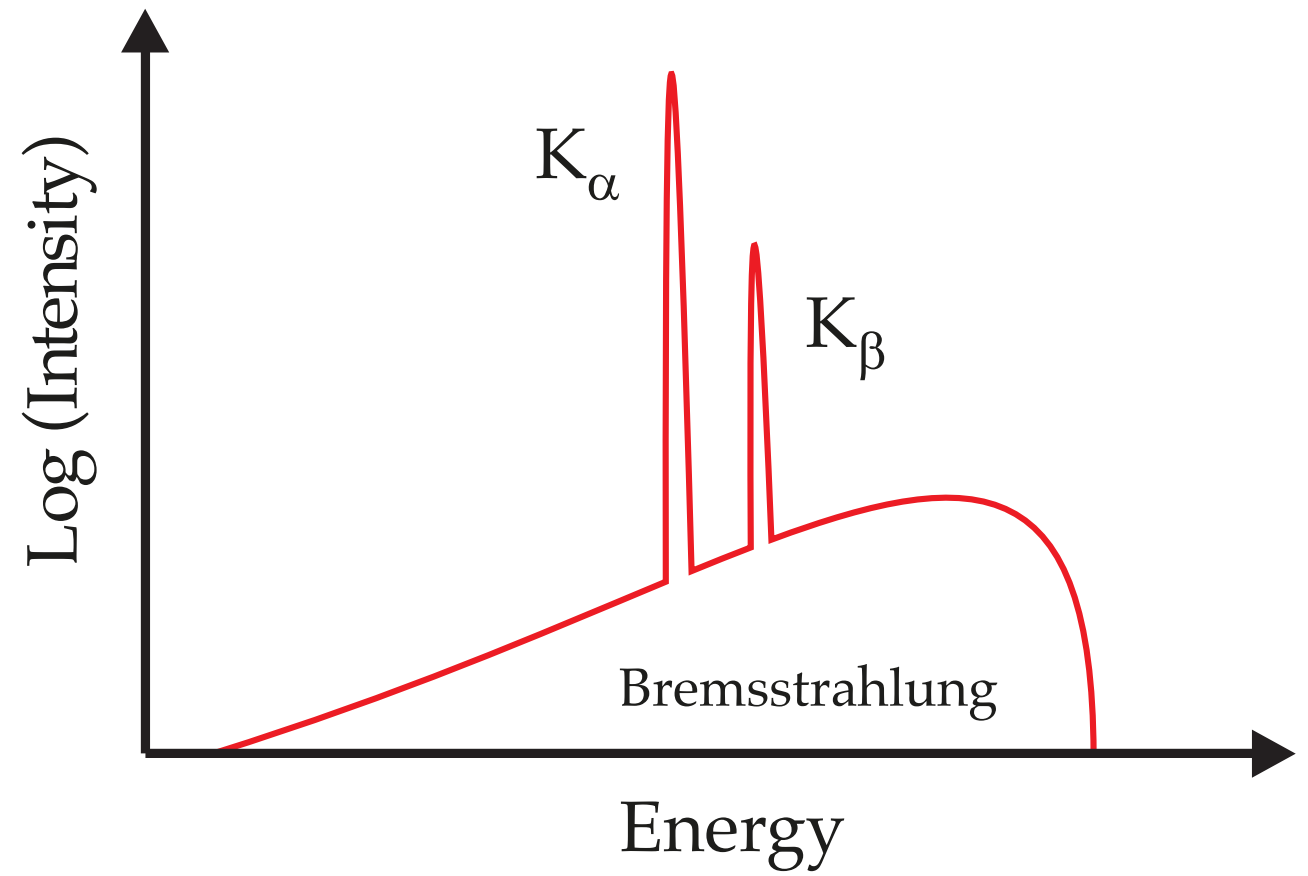

Figure 1: Characteristic and continuous radiation in the spectrum of an X-ray tube[13]

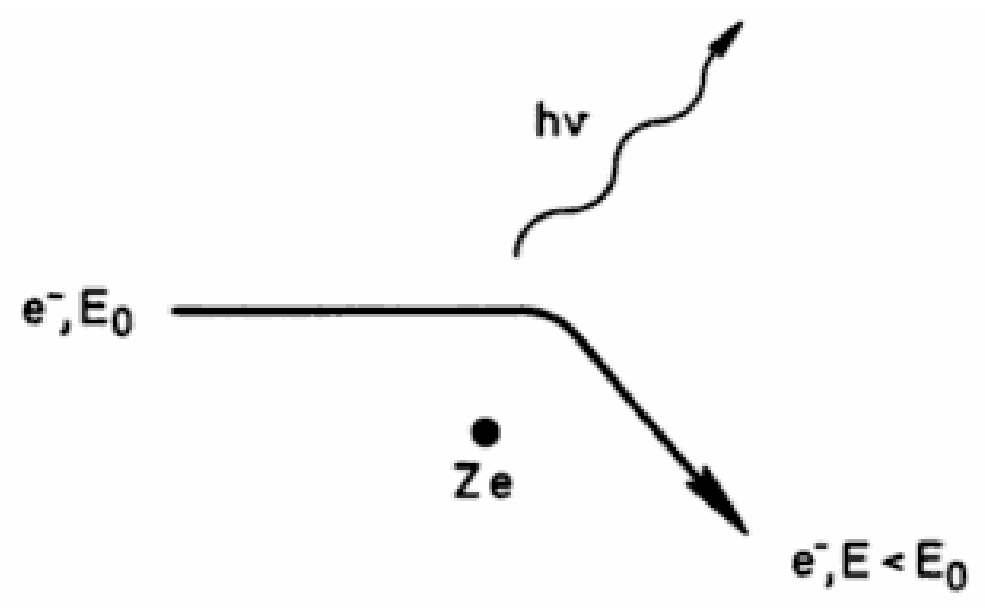

Figure 2: Production of Bremsstrahlung[14] 
duced in a continuous ("white") spectrum, up to a maximum frequency $v_{\text {max }}$ or minimum wavelength $\lambda_{\min }$ given by the Duane-Hunt law:

$$
\mathrm{eV}=\frac{h c}{\lambda_{\min }}=h v_{\max }
$$

where $e$ is the charge of the electron, $V$ is the excitation voltage, $h$ is the Planck constant and $c$ is the speed of light.

\subsubsection{Characteristic radiation}

The sharp, characteristic lines of the spectrum in Figure 1 on the other hand are caused by transitions of electrons between shells in an atom. As the energy levels of the shells are specific for an element and the energy difference of the transition is emitted in one photon, the produced radiation is characteristic for each element. The initial ionization of the atom, which is required for this transition to take place, can be caused by an electron or by incoming radiation of sufficient energy. Further details of the emission process will be discussed in section 2.2.1.

\subsection{Interaction of X-rays with Matter}

A beam of electromagnetic radiation passing through a material will be attenuated due to different interaction effects with the atoms of the material. More specifically $X$-rays with an energy below $100 \mathrm{keV}$, i.e. within the energy range typically used for $X$-ray spectroscopy, will interact with the electrons of the atoms mainly via the photoelectric effect (section 2.2.1) and to a lesser degree via scattering [15]. The latter component can be further distinguished into elastic and inelastic scattering, which will be described in sections 2.2 .3 respectively 2.2.2.

The transmitted intensity $I$ of a beam with inital intensity $I_{0}$ after passing trough a material of thickness $d$ and density $\rho$ is described by the Beer-Lambert law:

$$
I=I_{0} \exp \left[-\left(\frac{\mu}{\rho}\right) \rho d\right]
$$

where $\mu$ is called the linear attenuation coefficient and $(\mu / \rho)$ is called the mass attenuation coefficient. The attenuation coefficient $\mu$ can be divided into subcomponents, which represent the different interaction effects:

$$
\mu=\tau+\sigma_{e l}+\sigma_{\text {in }}
$$

where $\tau$ is the photoelectric, $\sigma_{e l}$ is the elastic and $\sigma_{i n}$ is the inelastic part of the 


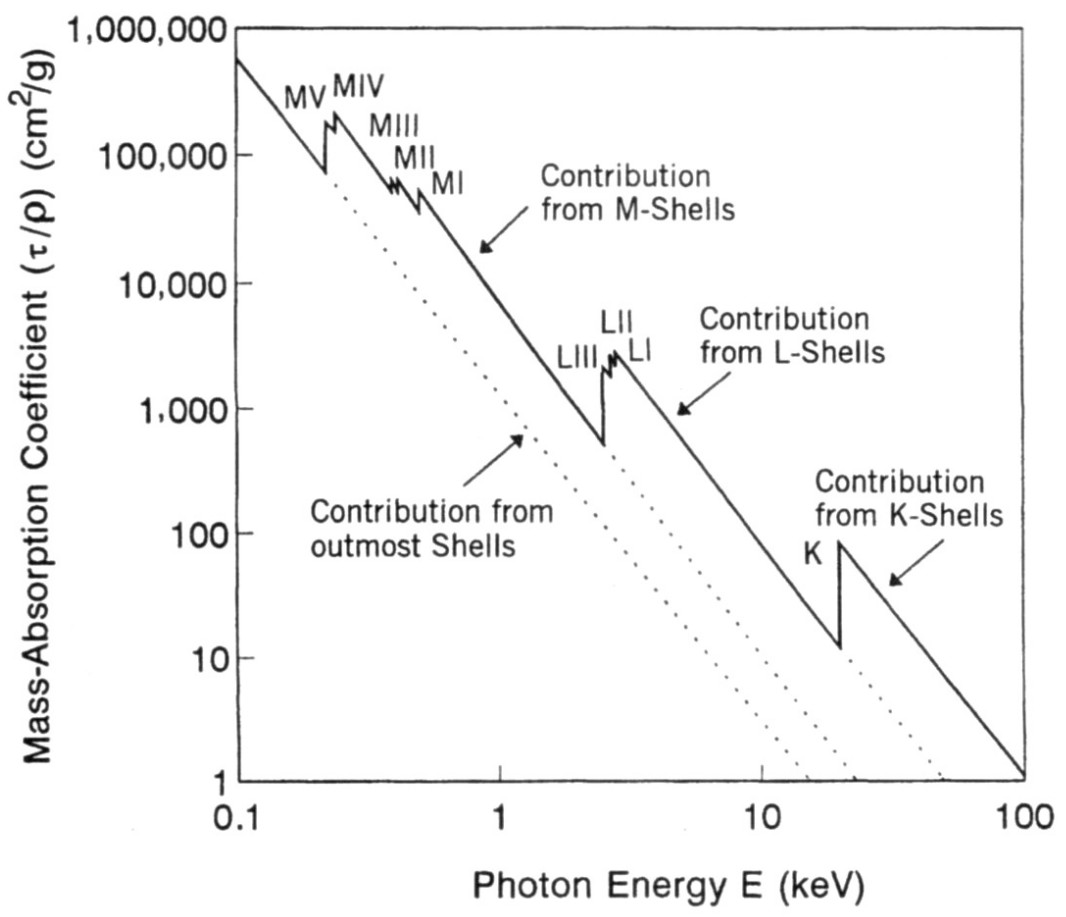

Figure 3: Total photoelectric mass-absorption coefficient for molybdenum.[15]

attenuation coefficient. These coefficients depend on the energy of the incident radiation and are specific for an element. The photoelectric absorption can even be split up further into contributions from different subshells (Figure 3).

\subsubsection{Photoelectric Absorption}

Electromagnetic radiation of sufficient energy, i.e. larger than the binding energy of the considered electron, can eject an electron from a shell in an atom and create a core hole. The incoming photon is annihilated in the process and ejects a photoelectron of energy

$$
E=E_{v}-E_{K}-W
$$

where $E_{v}$ is the energy of the incident radiation, $E_{K}$ is the binding energy and $W$ is the work function.

Subsequently the vacancy in the inner shell is filled by an electron from an outer shell and the difference in binding energies is either emitted as characteristic fluorescence radiation of energy

$$
E_{v}=E_{K}-E_{L}
$$




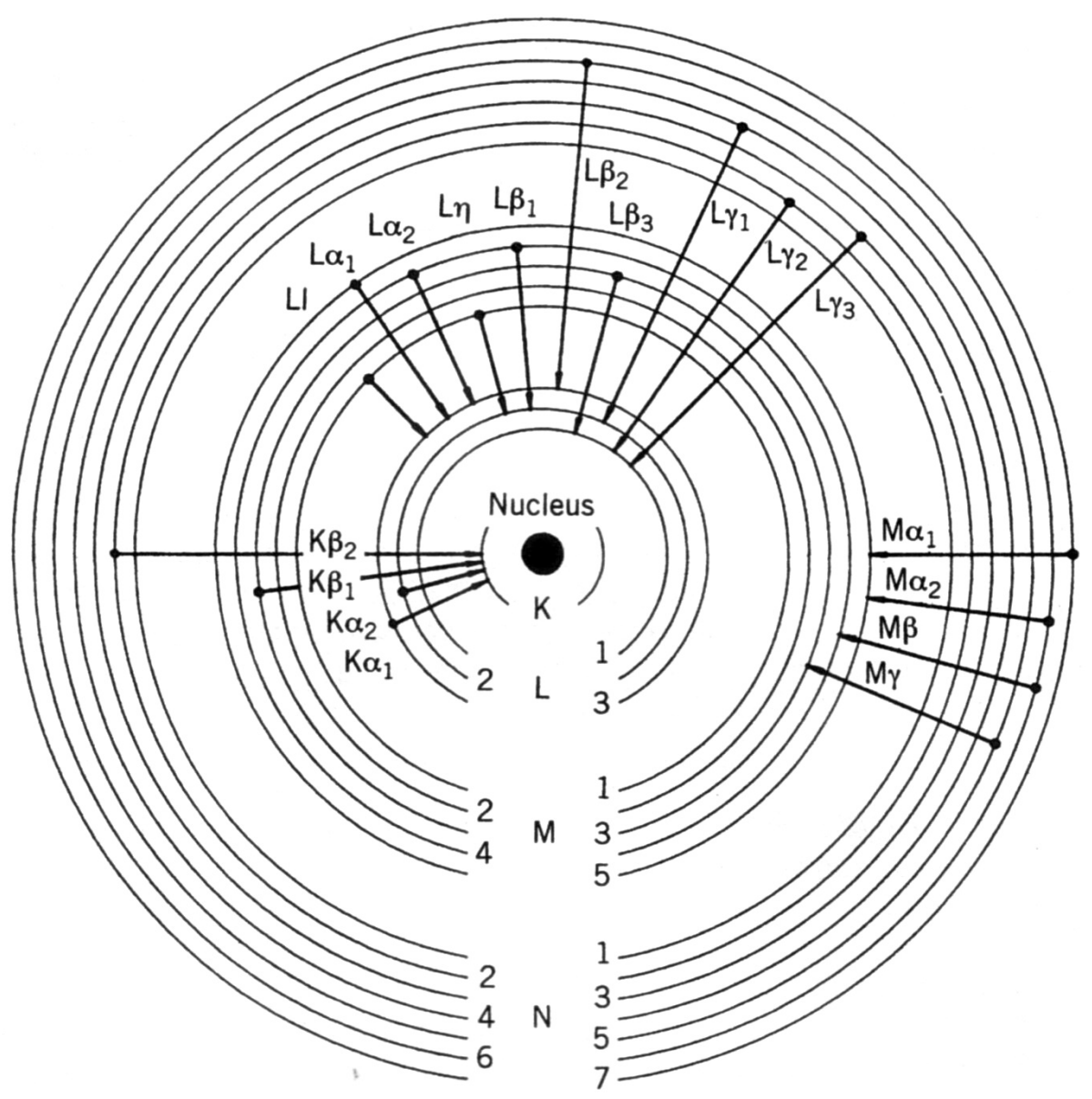

Figure 4: Electron transitions in a heavy atom and their names in Siegbahn notation.[15]

as an example for $\mathrm{K}$ and $\mathrm{L}$ shells or transferred to another electron, which is ejected from the atom as an Auger electron. A widely used nomenclature in X-ray spectroscopy for the identification of fluorescence lines is the Siegbahn notation, which is shown in Figure 4

The fluorescence yield, which gives the relative probability for the emission of fluorescence, is approximately described by

$$
\omega=\frac{Z^{4}}{A+Z^{4}}
$$

where $Z$ is the atomic number and $A$ is about $9 \times 10^{5}$ for the $\mathrm{K}$-series, $7 \times 10^{7}$ for the L-series and $1 \times 10^{9}$ for the M-series[15]. As shown in Figure 5 the probability of Auger electron emission increases dramatically for lighter elements, thus making X-ray fluorescence analysis difficult for these elements.

Figure 6 6 summarizes the photoelectric absorption, as well as the two competing processes of characteristic fluorescence and Auger electron emission. 


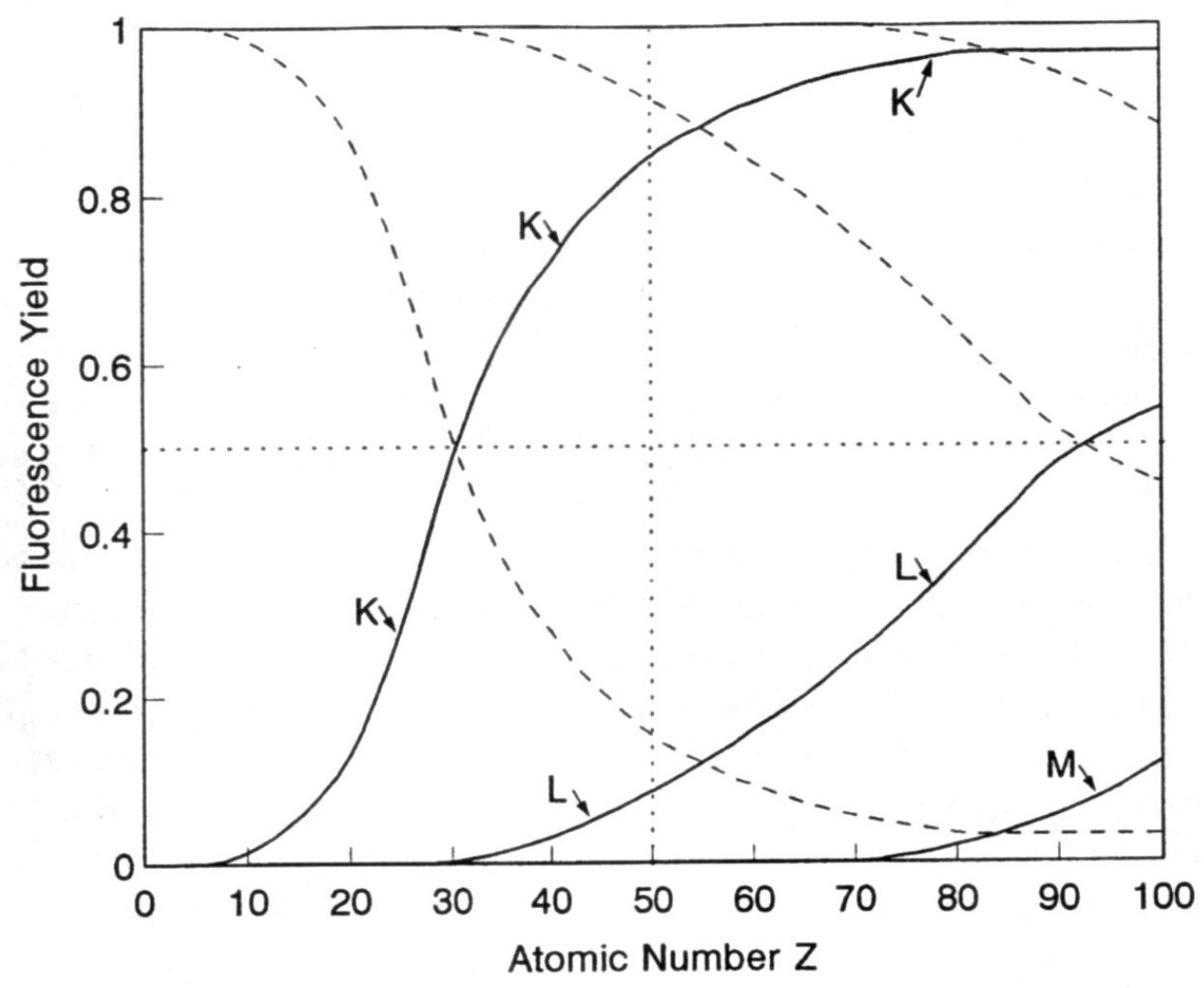

Figure 5: Fluorescence yield (solid line) and Auger electron yield (dashed line) [15]

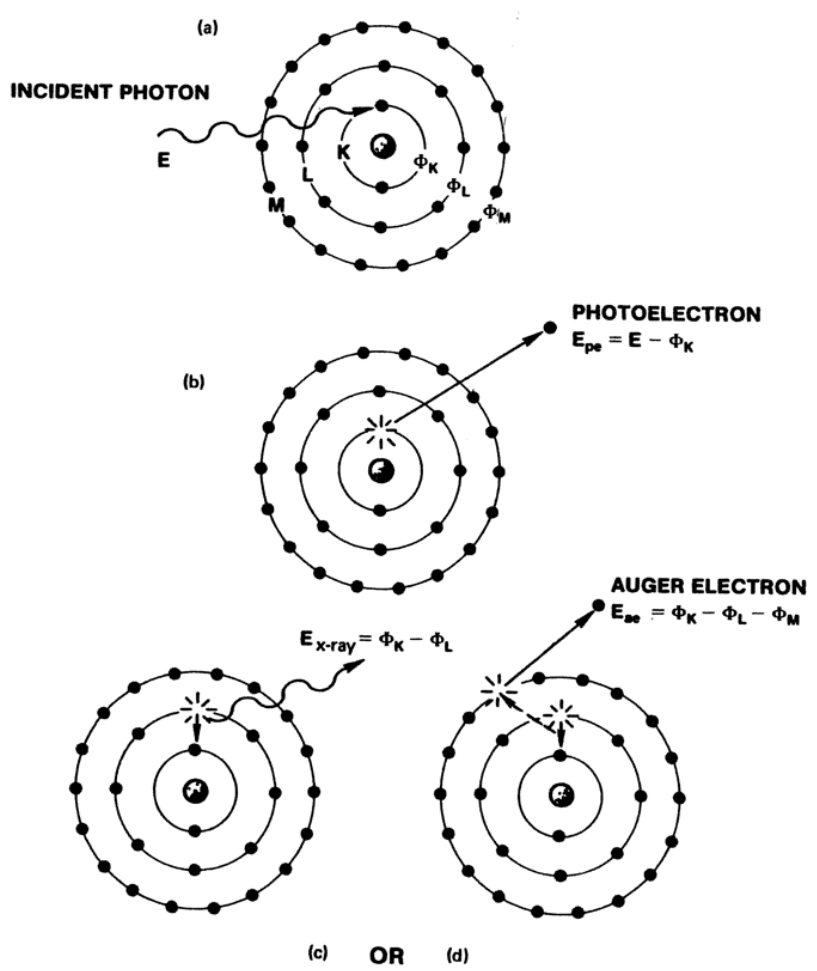

Figure 6: Overview of photoelectric absorption and the two emission processes. [16] 


\subsubsection{Inelastic / Compton Scattering}

If a photon interacts with a loosely bound outer electron or a free electron, and transfers part of its energy as well as part of its momentum to the electron, the process is called inelastic or Compton scattering. This scattering is always incoherent, i.e. there is no longer a fixed phase difference between the incoming and the scattered radiation. Due to the conservation of total momentum and energy, the relation between photon energy $E_{i}$ before and $E_{f}$ after the scattering by an angle $\psi$ can be calculated to

$$
E_{f} / E_{i}=\left[1+(1-\cos \psi) E_{i} / E_{e}\right]^{-1}
$$

where $E_{e}$ is the rest energy of an electron $(\sim 511 \mathrm{keV})$. This fraction depends on the initial energy, shows a maximum for forward scattering and a minimum for backward scattering. A calculation for the wavelength shows a wavelength shift

$$
\lambda_{f}-\lambda_{i}=\lambda_{C}(1-\cos \psi)
$$

where $\lambda_{C}$ is the Compton wavelength $(\sim 0.0024 \mathrm{~nm})$. This wavelength shift depends only on the deflection angle $\psi$. As can be seen in Figure 7, the intensity of the scattered radiation depends on the the initial energy $E_{i}$ and the scattering angle $\psi$ and shows a minimum around $90^{\circ}-100^{\circ}$.

\subsubsection{Elastic / Rayleigh Scattering}

In the case of elastic or Rayleigh scattering no energy is transferred between the incoming radiation and the bound electrons in the atom, nevertheless it causes a change in direction of the scattered radiation. For a single atom the intensity of the scattered radiation for unpolarized incident radiation of intensity $I_{0}$ and for a scattering angle $\theta$ can be given as

$$
I_{c o h}=I_{0} r_{e}^{2}\left[\frac{1+\cos ^{2} \theta}{2}\right] f^{2}\left(\frac{\sin \theta}{\lambda}, Z\right)
$$

where $f\left(\frac{\sin \theta}{\lambda}, Z\right)$ is the (tabulated) atomic form factor for incident radiation of wavelength $\lambda$ and an atom of atomic number $Z$ [18].

\subsection{Principles of X-ray Fluorescence Analysis}

The method of X-ray fluorescence analysis (XRF) is used as a non-destructive analytical technique in industrial as well as research applications. In many cases 


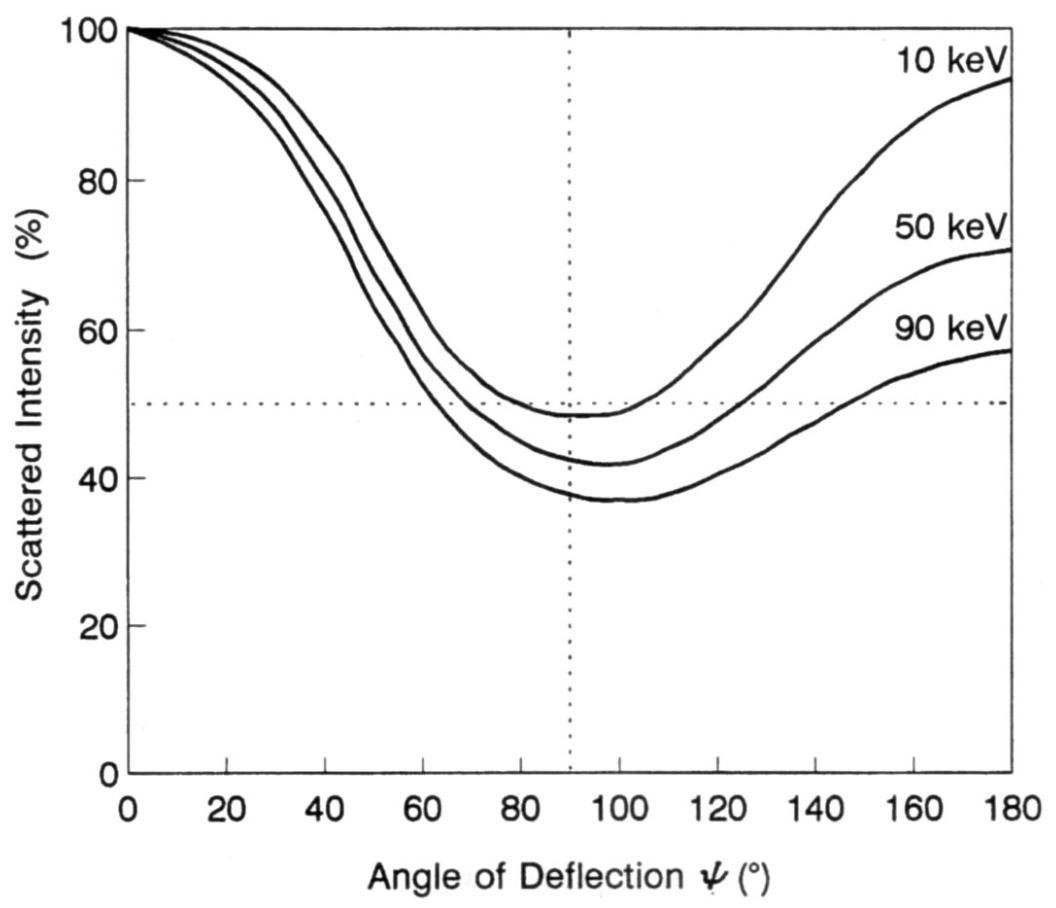

Figure 7: The normalized fraction of scattered intensity for Compton scattering.[17]

the use of XRF is relatively simple and straightforward, requiring no or only limited sample preparation. The analysis of light elements can be difficult and require special equipment like vacuum chambers, as the radiation emitted from low- $Z$ atoms is easily absorbed even in air and the fluorescence yield is very low.

XRF is based on the principles of photoelectric absorption and characteristic radiation as described in sections 2.2.1 and 2.1.2. The sample is excited by an X-ray beam from an X-ray source (section 2.3.1), absorbs part of the incident radiation and emits characteristic radiation, depending on the atoms present. An XRF spectrum gives qualitative and quantitative information on the chemical composition of a sample, as the intensity of the detected XRF-lines for an element is directly correlated to the number of atoms of this element in the sample.

\subsubsection{X-ray sources}

Common types of X-ray sources, which are used in XRF and related methods, include $X$-ray tubes and synchrotron radiation. While synchrotron sources provide superior brilliance, i.e number of photons emitted, their availability is limited to dedicated research centers and thus X-ray tubes are the most commonly used sources. Only the properties of X-ray tubes, which were used for this work, are discussed in this section.

A typical X-ray tube as shown in Figure 8 consists of a vacuum-sealed metal- 


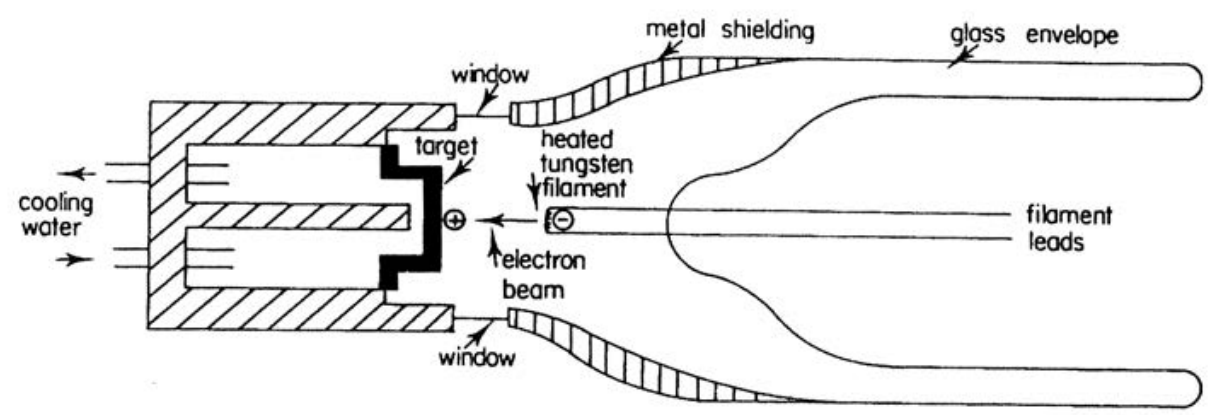

Figure 8: Sketch of an X-ray tube.[19]

glass assembly with anode and cathode in high vacuum and exit windows usually made of beryllium. A high voltage, typically in the range 10 to $100 \mathrm{kV}$ is applied between cathode and anode. The heated tungsten filament, which serves as a cathode, emits electrons, which are accelerated towards the anode by the high voltage. The electrons interact with the atoms of the anode material via processes described in section 2.1. Most of the electric input power is dissipated as heat, only about $0.1 \%$ are converted into radiation. The produced radiation spectrum contains continuous bremsstrahlung and characteristic fluorescence lines from the atoms of the pure-metal anode, e.g. chromium, copper, molybdenum or tungsten. In order to dissipate the heat, the anode is cooled by water or oil.

\subsubsection{Energy dispersive X-ray detectors}

Energy dispersive XRF (EDXRF) uses solid state, semi-conductor based detectors to acquire and process a wide range of the $\mathrm{X}$-ray fluorescence spectrum, which is emitted by the sample, virtually simultaneously. The latest generation of these detectors, the Silicon Drift Detector (SDD) in combination with a Digital Pulse Processor (DPP) is able to process count rates up to 1000000 counts per second and achieve an energy resolution down to $130 \mathrm{eV}$. The SDD uses concentrical stripes of varying potential with an electron potential minimum in the middle of the wafer (Figure 9). In this way electrons, which are created by an incoming and absorbed photon in the depleted volume of the detector, will be collected at the readout anode. This principle of sideward depletion proposed by Gatti and Rehak [21] offers the advantage, that the value of the anode capacitance is small in comparison to a standard diode and practically independent of the total area of the device. This in turn translates to less noise in the signal, as the rise time is shorter and the signal itself, which is proportional to the energy 


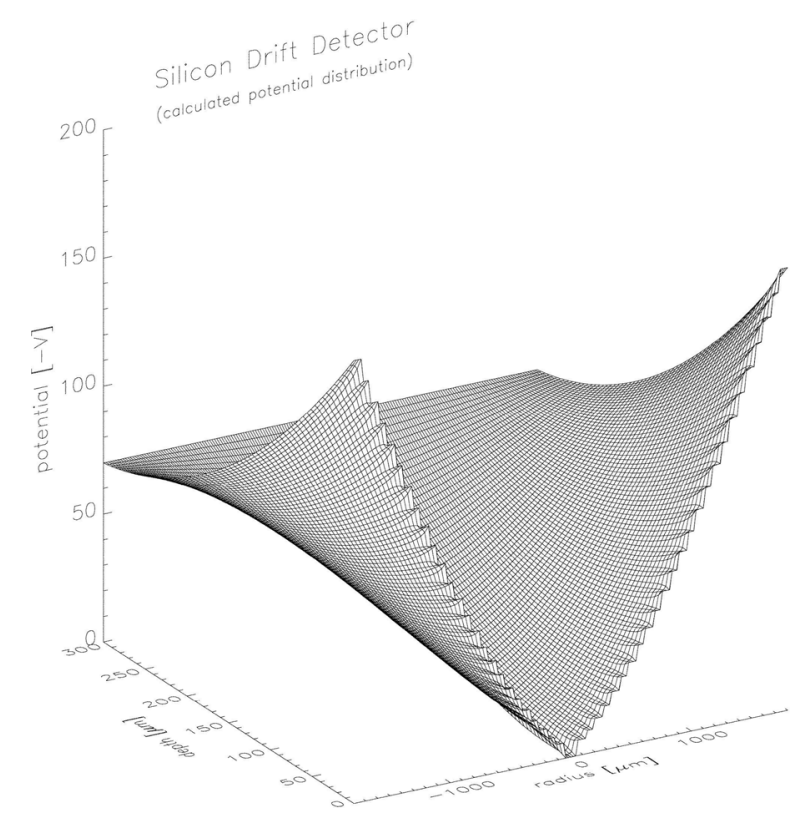

Figure 9: Potential energy distribution in a SDD.[20]

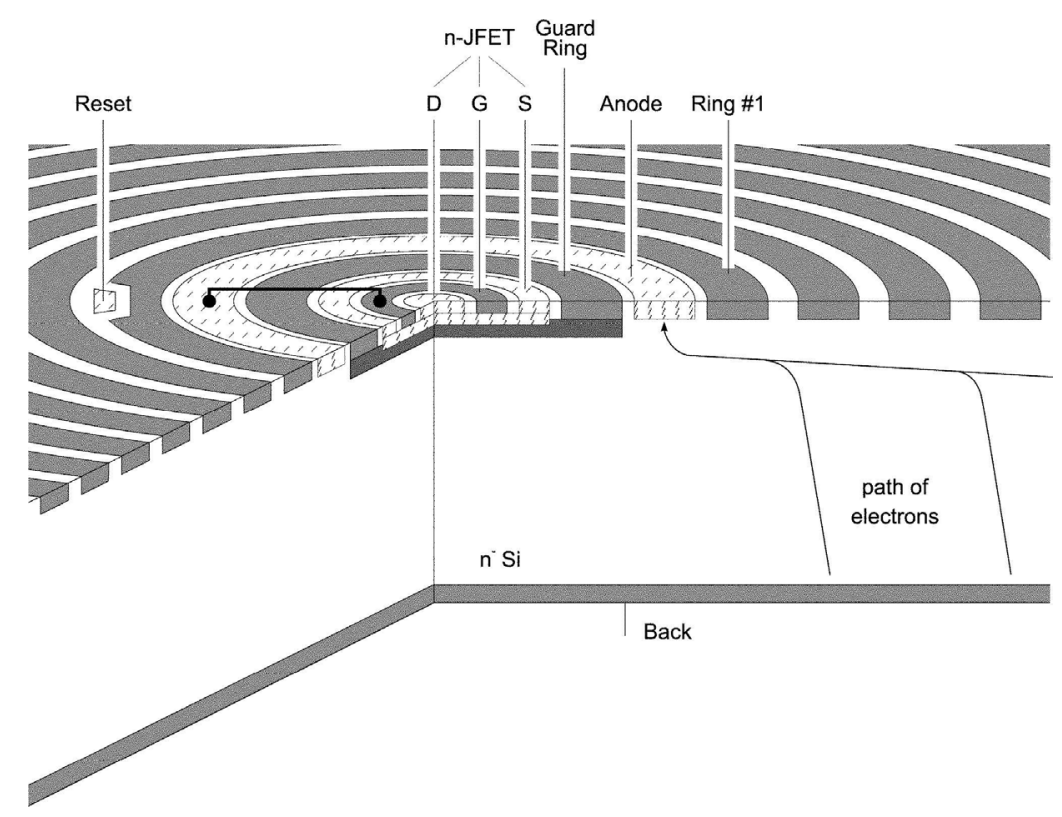

Figure 10: Central area of a cylindrical SDD with integrated amplifier.[20] 
of the absorbed photon, has a bigger amplitude [20]. In most cases the design also includes an amplifying field effect transistor (FET) directly connected to the anode on the detector chip as shown in Figure 10. This also helps to reduce noise as the capacitance of the detector-amplifier system is minimized.

Typical shaping times, i.e. the time required to process the signal produced by a single photon and also approximately the time during which no further signal may arrive, are in the range of some microseconds for SDD systems. These times also offer the best energy resolution, but the time can be reduced to some hundred nanoseconds, if energy resolution is not of concern.

As a further advantage a SDD only requires cooling by a Peltier cooler, which can be easily integrated into the detector package and requires no coolant refilling like a liquid nitrogen cooled detector.

\subsection{Principles of Grazing Incidence X-ray Fluorescence}

\subsubsection{Total Reflection X-ray Fluorescence}

Total reflection X-ray fluorescence analysis (TXRF) is in principle EDXRF in a somewhat exotic geometry, but offers distinct advantages in several applications. A typical TXRF setup, which is also usable for Grazing Incidence X-ray Fluorescence analyis (GIXRF), is shown in Figure 11.

The incident beam hits, in the case of GIXRF, the sample or, in the case of TXRF, the sample carrier, which should be smooth and polished in order to be reflecting, at a very small angle. If the angle between reflector and beam is small enough, i.e. below critical angle $\alpha_{\text {crit }}$, total external reflection of the X-ray beam will occur. This is due to the fact that the refractive index in the $X$-ray range can

\section{TXRF/GIXRF setup}

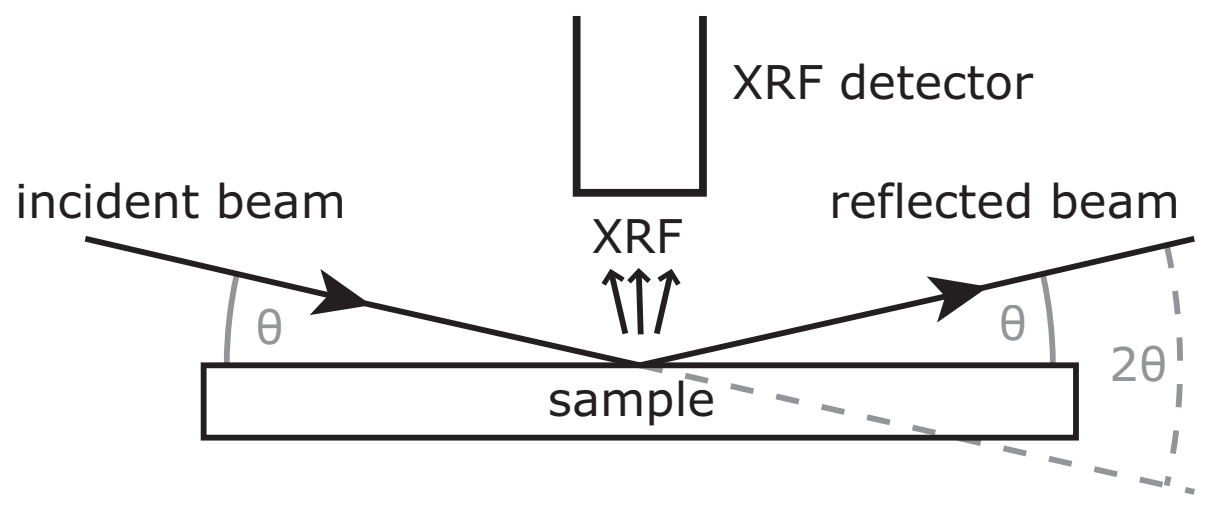

Figure 11: Schematic illustration of a TXRF or GIXRF setup. 
be written as a complex quantity [15]:

$$
n=1-\delta-i \beta
$$

The imaginary component $\beta$ is a measure of the attenuation and for radiation of wavelength $\lambda$ is defined by [15, 22]

$$
\beta=\frac{\lambda}{4 \pi}\left(\frac{\mu}{\rho}\right) \rho=\frac{\rho N_{a} r_{e} \lambda^{2}}{2 \pi A} f_{2}^{0}(\omega)
$$

where $(\mu / \rho)$ is the mass attenuation coefficient, $\rho$ is the density, $N_{a}$ is Avogadro's number $\left(6.022 \times 10^{23}\right.$ atoms $\left./ \mathrm{mol}\right), r_{e l}$ is the classical electron radius $(2.818 \times$ $\left.10^{-13} \mathrm{~cm}\right), A$ is the atomic weight and $f_{2}^{0}(\omega)$ is the imaginary part of the atomic scattering factor in the forward direction. In a similar way the real part $\delta$, which measures the deviation of the real component from unity, is given by [22, 23]

$$
\delta=\frac{\lambda^{2}}{2 \pi} r_{e} \rho_{e}=\frac{\rho N_{a} r_{e} \lambda^{2}}{2 \pi A} f_{1}^{0}(\omega)
$$

where $\rho_{e}$ is the electron density (including dispersion) and $f_{1}^{0}(\omega)$ is the real part of the atomic scattering factor in the forward direction. The values of $\delta$ are quite small (between $2 \times 10^{-7}$ and $5 \times 10^{-3}$ ), but nevertheless result in a real part of the refractive index smaller than unity.

The critical angle of total reflection can be approximated by the relation

$$
\alpha_{\text {crit }} \approx \sqrt{2 \delta}
$$

which gives values between $0.04^{\circ}$ and $0.6^{\circ}$ for media and X-ray energies as typically used in TXRF.

The advantages of TXRF can be summarized as follows [15, 24, 25, 26] :

- Increased (double) excitation of the sample due to penetration by primary and reflected beam.

- Reduced background as a result of the very limited penetration into the sample carrier.

- Increased solid angle of detection as the detector can be positioned very close to the sample.

- Only small sample volume is required

- Detection limits with X-ray tubes in the pg-range [27, 28]. 


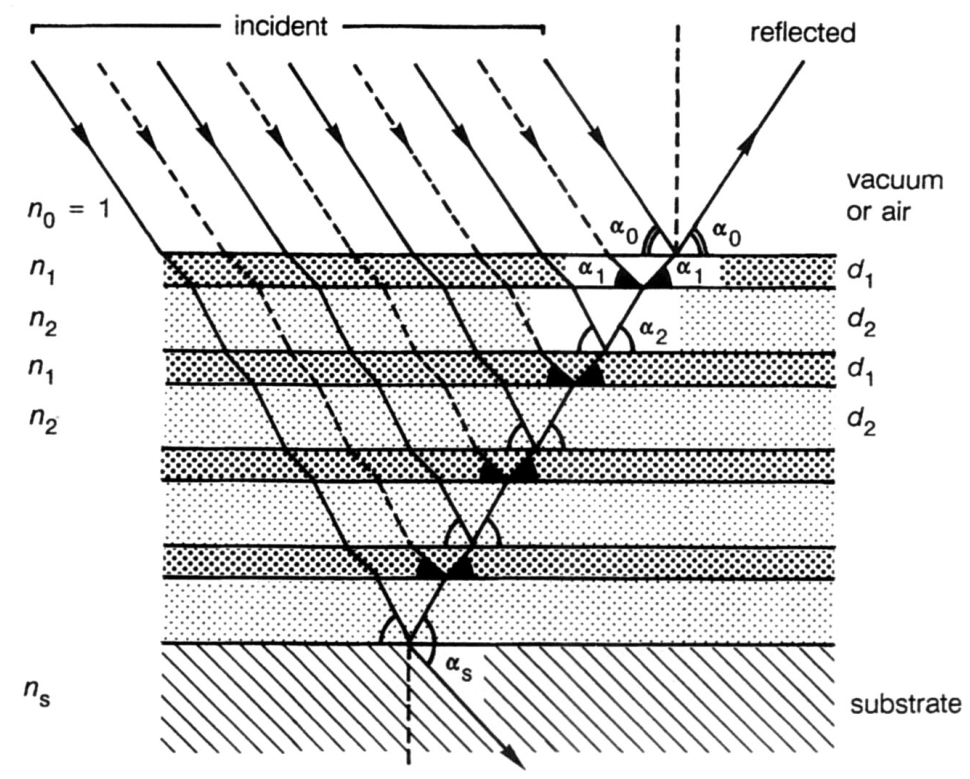

Figure 12: A periodic multilayer consisting of $\mathrm{N}$ bilayers with a period $d=d_{1}+d_{2}$ and at total thickness $D=N d$ deposited on a thick flat substrate. The layers with index 1 are the reflectors; the layers with index 2 are the spacers. After coherent scattering, several reflected X-ray beams can interfere with each other.[15]

\subsubsection{Monochromatization of X-rays}

As the refractive index and thus the critical angel is energy/wavelength dependent, the primary beam should only consist of a limited band of wavelengths, in order to obtain good results with TXRF and GIXRF. This monochromatization of the beam can be achieved trough different devices like crystals, absorption filters, total reflection mirrors and synthetically produced multilayers. In this section the multilayer monochromator, which was used during this work, is described.

A multilayer monochromator consists of a periodic succession of two layer materials with constant bilayer thickness $d=d_{1}+d_{2}$. The two materials are carefully chosen, with one acting as a "spacer" with low absorption and the other (the "absorber") should offer a high contrast in refractive index. Typical pairs of material are Tungsten/Carbon or Molybdenum/Silicon.

The working principle of a multilayer can be easily understood by modeling the incoming radiation as a plane wave with wavelength $\lambda$. At each layer boundary the wave is refracted and reflected. If the path difference for reflections from two neighboring reflectors is an integer of $\lambda$, constructive interference will occur and the intensity of the respective wavelength is reinforced. Figure 12 illustrates the respective beam paths. 


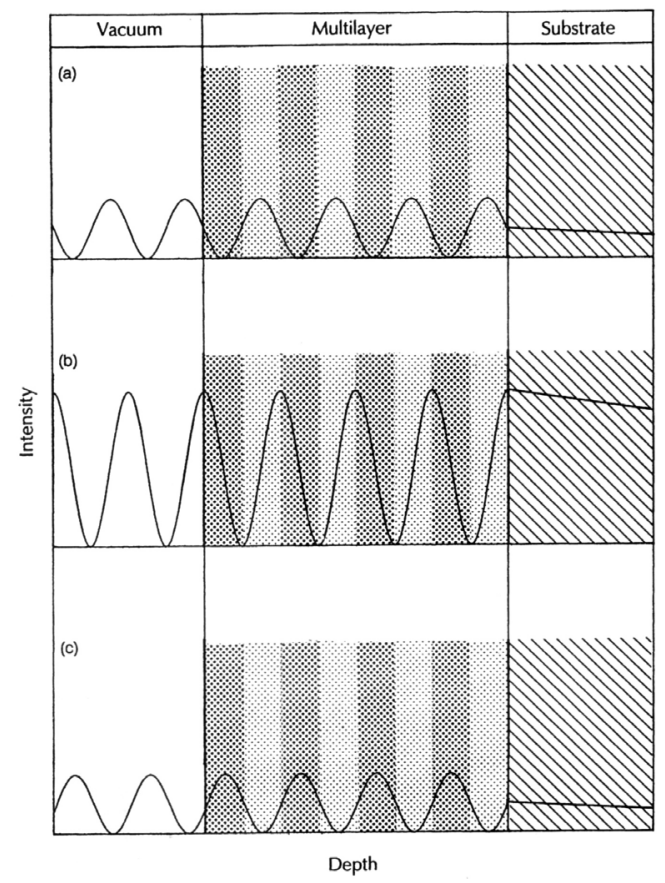

Figure 13: Standing waves within a multilayer consisting of a stack of four bilayers for the case of Bragg reflection. The reflectors are dotted darker; the spacers, dotted ligher. Case (a) represents an angle just below the Bragg angle, which is exactly reached in case (b), while case (c) represents an angle just above the Bragg angle. In all three cases, the wave pattern has the periodicity of the multilayer itself, but the antinodes jump from the spacers in case (a) to the reflectors in case (c). The Bragg angle with the highest reflectivity is reached in case (b).[15]

Neglecting absorption and refraction, this characteristic can be described by Bragg's law for a multilayer of spacing $d$ and X-rays of wavelength $\lambda$

$$
2 d \sin \alpha_{m} \approx m \lambda
$$

where $m$ is an arbitrary integer and $\alpha_{m}$ is the glancing angle of incidence and reflection. Therefore a specific energy range, e.g. characteristic fluorescence from the anode material of the X-ray tube, can be selected from the emitted spectrum by using the proper incident and reflection angle at the multilayer.

By the same consideration on incoming and reflected wave, one can understand the X-ray Standing Wavefield (XSW), which is formed above the substrate due to constructive and destructive interference within the multilayer structure and above (Figure 13).

\subsubsection{Grazing Incidence X-ray Fluorescence}

In GIXRF the primary intensity in the sample appears as an evanescent wave field or as a standing wave field with locally dependent fluctuations (Figure 14). 


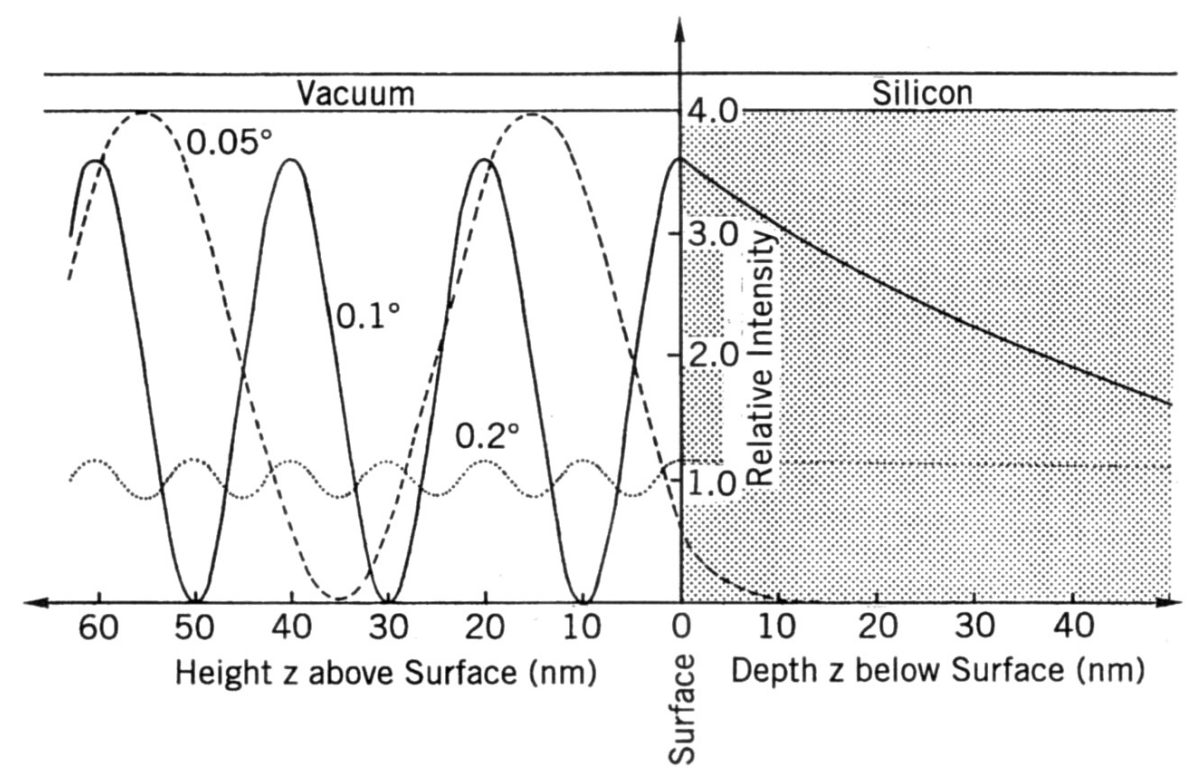

Figure 14: X-Ray intensities above (within the standing wave field) and below a thick Si-flat calculated for different angles of incidence. It can be seen that the dependence of the distance $D$ between nodes and antinodes is a function of the incident angle. Inside the medium, the intensity decreases as a function of the refraction angle.[15]

The intensity of the fluorescence radiation emitted by atoms which are excited by these fields is direct proportional to the wave field intensity. Therefore the fluorescent signal emitted by a sample refers to the varying field intensity of the standing or evanescent wave field within the sample. Moreover it additionally provides information on the elemental composition of the sample. As the distribution of nodes and antinodes of the standing wave field within the sample is a function of the incident angle of the primary radiation, the (consequently angle dependent) fluorescence signal can be used for nondestructive depth profiling of layered structures.

For the analysis of thin-film-like samples ("nanofilms") on a reflecting substrate the analyte has to be positioned within the standing wave field in front of the substrate. Generally two cases have to be considered for thin films: nonreflecting layers and reflecting layers on the surface of the substrate. Considering the first case it is assumed that non-reflecting layers should not disturb the primary wave field in front of the substrate significantly. Total reflection of the primary beam does not happen at the nanofilm itself but only at the substrate. The sample is excited to fluorescence and the emitted intensity is proportional to the primary field intensity. The intensity of this field with nodes and antinodes can be calculated with [15]: 


$$
I(\alpha, z)=I_{0}\left[1+R(\alpha)+2 \sqrt{R(\alpha)} \cos \left(\frac{2 \pi z}{a-\phi(\alpha)}\right)\right]
$$

where $I_{0}$ is the intensity of the primary beam, which is assumed to be constant. $R$ is the reflectivity of the substrate, $z$ is the height above the surface of the substrate and the argument of the cosine represents the phase difference of the incoming and reflected waves (the travel distance $2 \pi z / a$ and a phase shift $\phi$ ). This phase shift occurs only in the region of total reflection $\left(\phi=0\right.$ for $\left.\alpha>\alpha_{\text {crit }}\right)$ and is given by:

$$
\phi(z)=\arccos \left[2\left(\frac{\alpha}{\alpha_{\text {crit }}}\right)^{2}-1\right]
$$

Considering a thin film of thickness $t$ the totally emitted intensity of the sample is obtained by integrating equation 17 from $z=0$ to $z=t$. An example of this calculation for different thicknesses $t$ is shown in Figure 15. In the angular region of total reflection strong oscillations occur for thicknesses smaller than about $100 \mathrm{~nm}$ because only a few nodes and antinodes of the standing wave field penetrate the nanofilm.

In the next paragraph reflecting layers are discussed. They are assumed to be flat, smooth and of constant thickness (about $0.2-100 \mathrm{~nm}$ ) over the entire surface of the substrate. The angle dependent fluorescence emitted by such layers excited by X-ray can be calculated by means of fundamental parameters. First the primary intensity within the layer has to be computed as a function of penetration depth. Then excitation to fluorescence, absorption and enhancement must be included in the calculation. Eventually the fluorescence intensities have to be integrated over the layer thickness. The calculations are somewhat lengthy and are not presented here - an overview can be found inter alia in [15] or [6]. Figure 16 shows the results of calculations for the angle dependent fluorescence intensity emitted from a layer on Silicon substrate.

From Figure 16 it can be seen, that the curves of the ultra-thin Co layers are similar to those of non-reflecting layers. The maxima of those layers are located at the critical angle of Silicon (1.78 mrad for $17.5 \mathrm{keV}$ excitation energy). These maxima are shifted towards the critical angle of Co (3.35 mrad for $17.5 \mathrm{keV}$ excitation energy) for layers thicker than $10 \mathrm{~nm}$. The reason is that for nanometerthin Co-layers the radiation penetrates the film and total reflection occurs at the Si-substrate exclusively. For films thicker than about $10 \mathrm{~nm}$ the curves show oscillations of intensity above the critical angle of Co. These fluctuations are correlated to the Kiessig maxima and minima of the reflectivity of the layered 


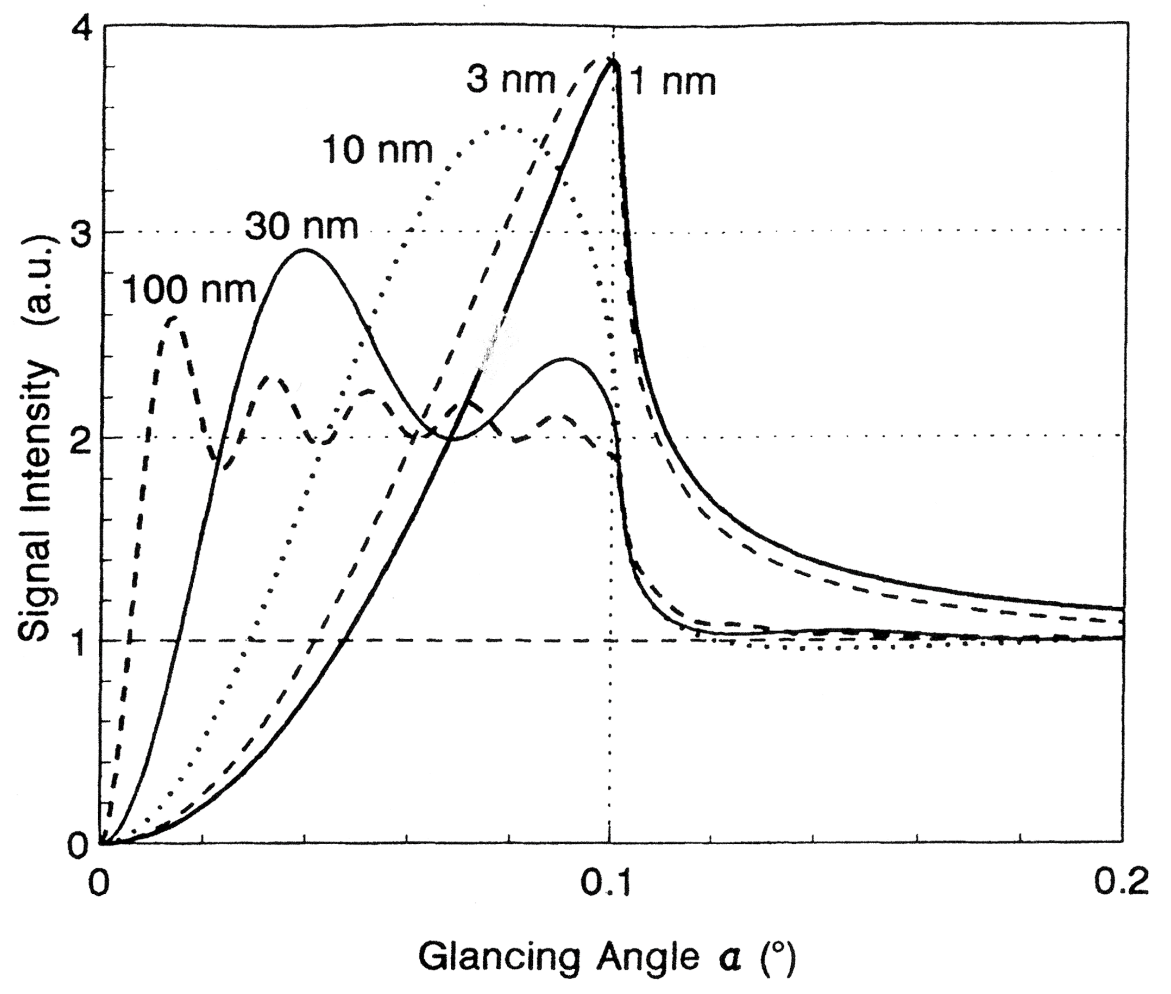

Figure 15: Fluorescence signals of thin (non-reflecting) films on a silicon reflector excited by Mo$\mathrm{K} \alpha$ radiation. The intensities have been calculated as a function of incident angle $\alpha$ for different thicknesses but equal mass of the samples.[15]
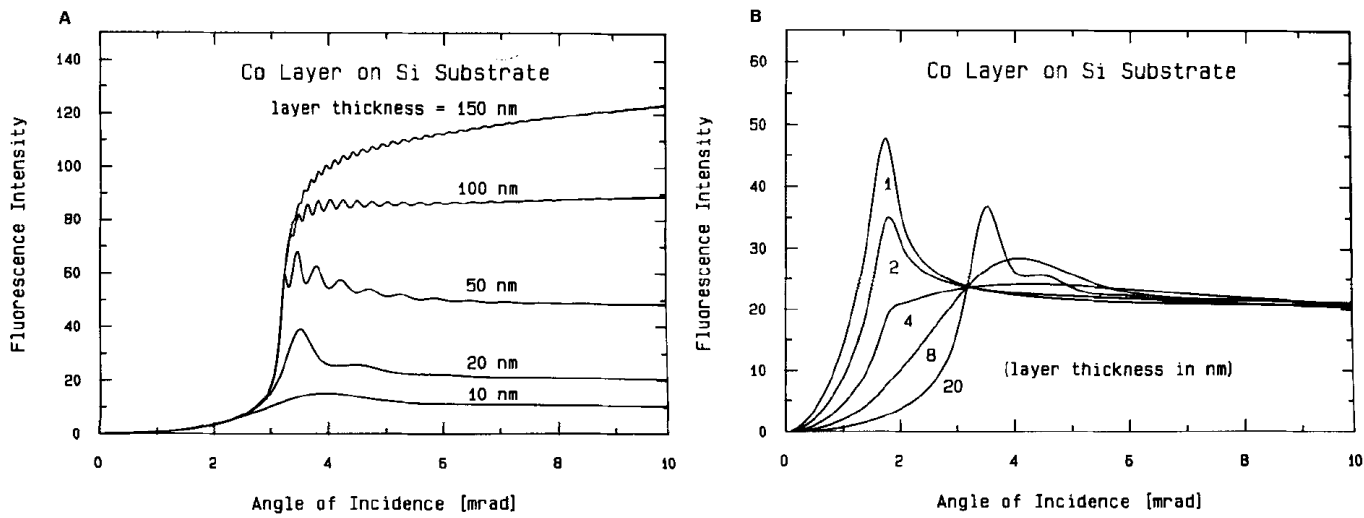

Figure 16: (A) Calculated fluorescence intensities as a function of incident angle for different layer thicknesses of Co on a silicon reflector. Excitation energy: Mo-Ka (17.5 keV). (B) as in (A), but fluorescence radiation normalized to the intensities at large angles of incidence.[29] 
sample [15, 6].

Finally for determination of the layers present in a given sample the measurement data, obtained by varying the incident angle of primary radiation across the critical angle of total reflection of the substrate and - in case of reflecting layers - the layer material, are evaluated by an iterative fitting procedure on the basis of modeling calculations. This procedure was developed and described by several authors [4, 30]. 


\section{Instrumentation}

\subsection{X-ray source}

In order to carry out GIXRF measurements, the primary beam should have a low height (with respect to the reflector surface) in the range of some $10 \mu \mathrm{m}$ and a large width (about $10 \mathrm{~mm}$ ) as the detector window is usually in this range [15]. This can be realized by using an X-ray tube with a line focus and an aperture system restricting the beam in height.

According to the demands on the angle adjustment equipment the divergence of the beam should not be worse than the angular resolution of a scan (that means better than $0.01^{\circ}$ ). This has to be achieved by a slit system optimized for the setup.

It is necessary to use a monochromator because only a monochromatic incident beam produces the angle dependent intensity profiles that are distinctly determined by the layered samples[15]. Therefore a strong spectral peak of the primary spectrum should be selected for excitation by a suitable monochromator - typically a multilayer monochromator acting as Bragg reflector. Spectral parts like other peaks than the selected one are unwanted because they would disturb the correlation between profile and layered sample.

In the present design the primary source is a $3 \mathrm{~kW}$ Mo anode X-ray tube with a focal spot of $0.04 \times 12 \mathrm{~mm}^{2}$ (long fine focus tube). In order to achieve low radiation background in the fluorescence spectra along with suitable photon flux for thin-film analysis, the primary $\mathrm{X}$-ray beam is monochromatized to $17.44 \mathrm{keV}$ (Mo-K $\alpha$ ) by means of a multilayer monochromator. To avoid unwanted effects due to totally reflected $X$ rays at the multilayer surface a $50 \mu \mathrm{m}$ diaphragm is placed between multilayer and sample. The proper Bragg angle between incident radiation and multilayer surface is adjusted by positioning the $\mathrm{X}$-ray tube using a stepper motor providing a linear translation of the tube versus a fixed slit. The movement is done until a maximum in the Mo-K $\alpha$ intensity is reached.

\subsection{Vacuum chamber and sample stage}

For the investigation of thin layers angle dependent measurements have to be performed to obtain intensity profiles. Therefore it is necessary to be able to vary the angle of incidence between $0^{\circ}$ and about $2^{\circ}(34.9 \mathrm{mrad})$ depending on 


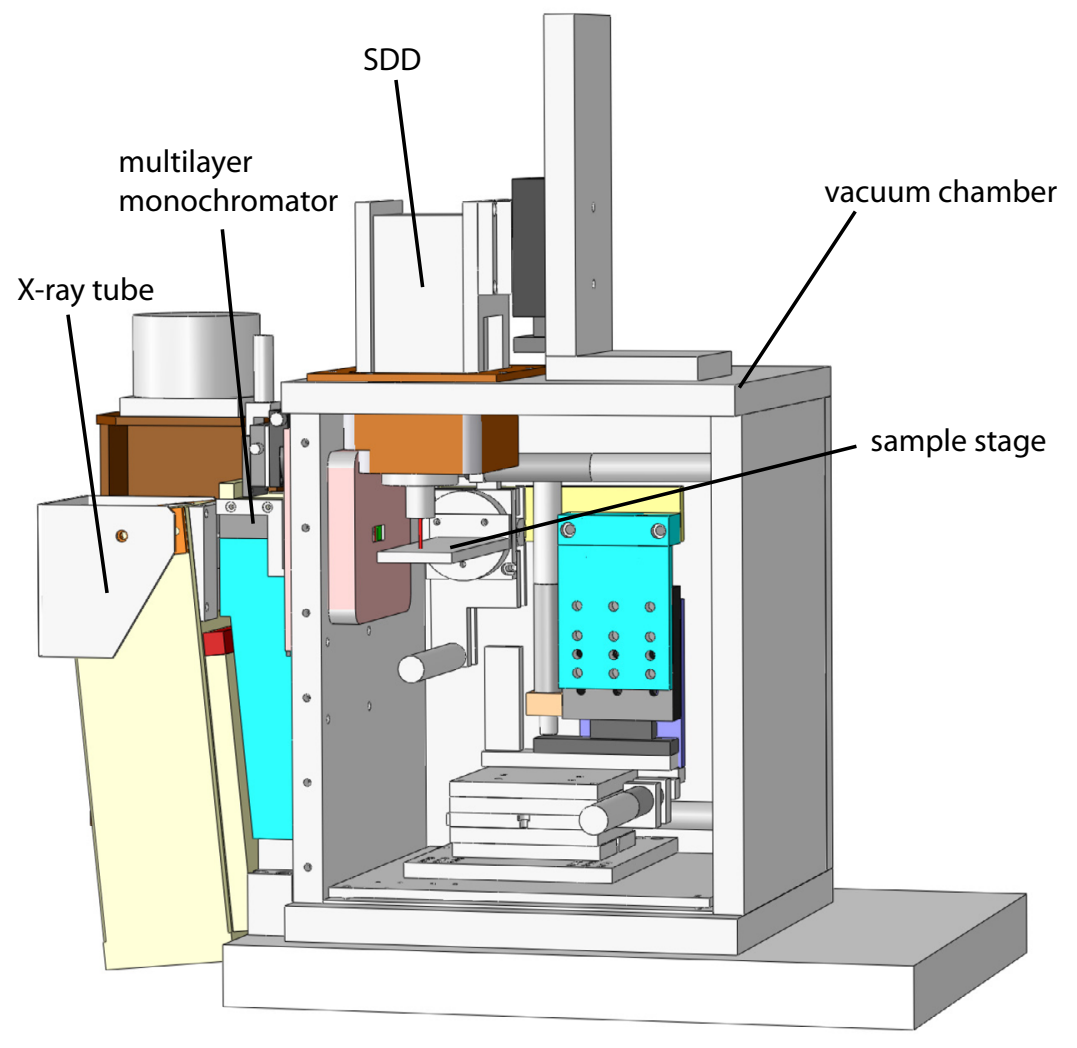

Figure 17: Design and main components of the GIXRF spectrometer

the used substrate. To get reasonable angular resolution of the intensity profiles the step-size of an angle scan should be below $0.01^{\circ}(0.174 \mathrm{mrad})$. Furthermore the adjusted angle has to be controlled with high accuracy (better than $0.005^{\circ}$ $(0.087 \mathrm{mrad}))$ to enable a reliable quantification of the intensity profiles[15].

A schematic view of the GIXRF prototype spectrometer and its degrees of freedom are shown in Figures 17 and 18 . Supplemental to tilting the sample by a defined angle PHI for recording an intensity profile, some other movements of the sample and the slit system are necessary for optimum adjustment. As already mentioned the slit after the monochromator defines the beam height and has to be adjusted (tilting and height adjustment) referring to the line focus of the tube and the properties of the monochromator system. This has to be done in order to obtain maximum intensity, well defined dimensions (height and width) and low divergence of the beam at the sample position. After that the beam illuminates a well defined volume in front of the detector (typically 'below' the detector for 'down looking' systems) and the sample has to be moved into this region. This is achieved by advancing the reflector towards the detector $(Z)$ until the sample is hit by the X-ray beam. Additionally, tilting of the sample (THETA) may be necessary to align it parallel to the beam. Movement of the sample in 


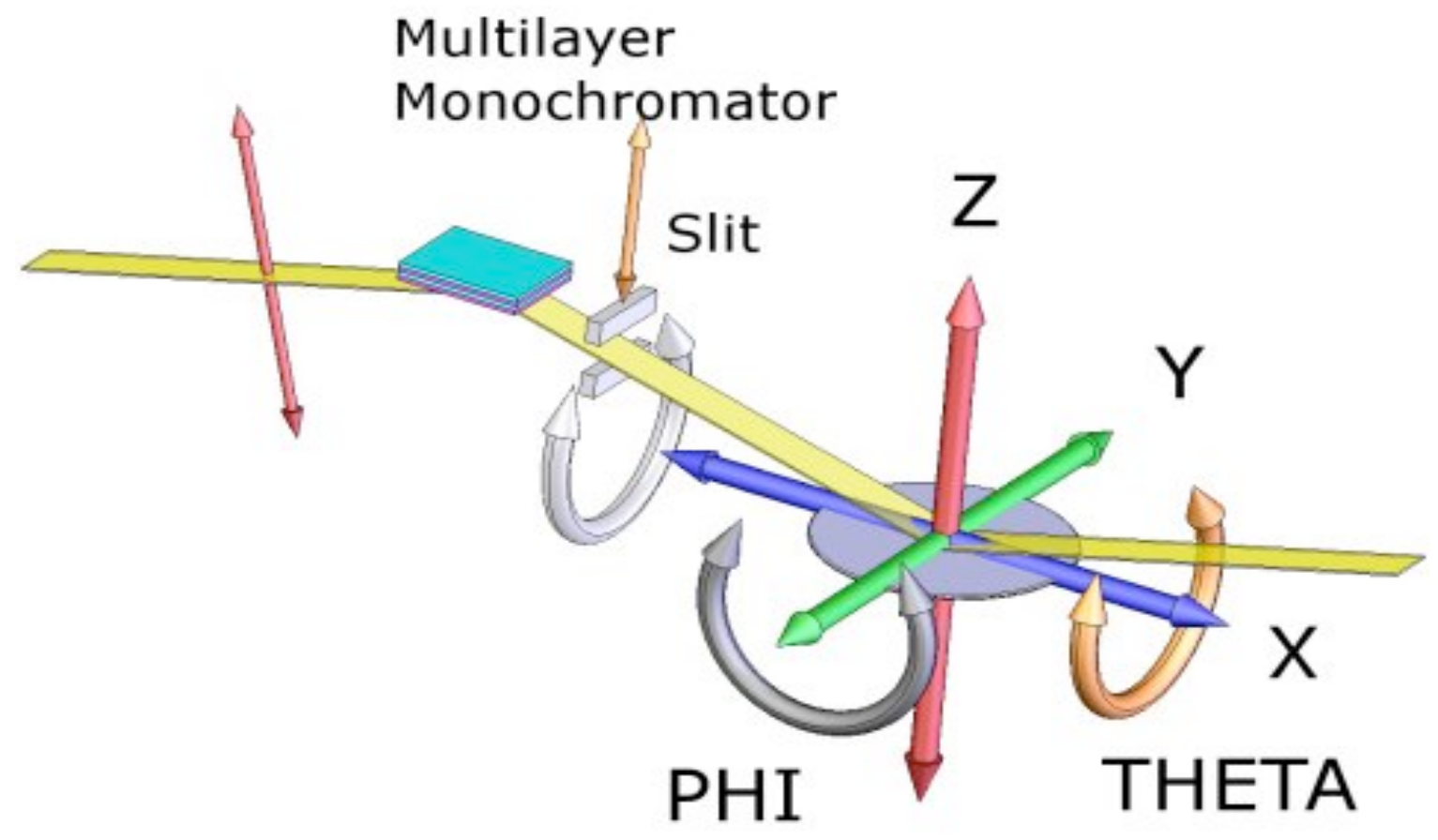

Figure 18: Sketch of the beams optical path and the degrees of freedom in a GIXRF setup.

the Upstream/Downstream position $(X)$ may be necessary, in order to move the center of rotation below the center of the detector. Figure 19 shows the influence of a misaligned center of rotation on the measurement.

To avoid scattering of the primary $X$-rays in air and to minimize the absorption of low energetic fluorescence X-rays (e.g. Si emits $1.7 \mathrm{keV}$ radiation and is used to normalize for quantification and dose determination) on their way to the detector, the whole setup is placed in a vacuum chamber with an overall size of $300 \times 300 \times 340 \mathrm{~mm}^{3}$. In order to perform all movements necessary for adjustment and measurement of the sample, the sample stage consists of 3 linear stages with a travel range of $25 \mathrm{~mm}$ each and one rotation stage allowing to perform angle scans in the range of \pm 0.28 rad with a minimum increment of $1.35 \mu \mathrm{rad}$. The stage can be tilted around the beam-axis to align the sample parallel to the beam.

Figure 20 shows an image of the interior of the GIXRF spectrometer. 


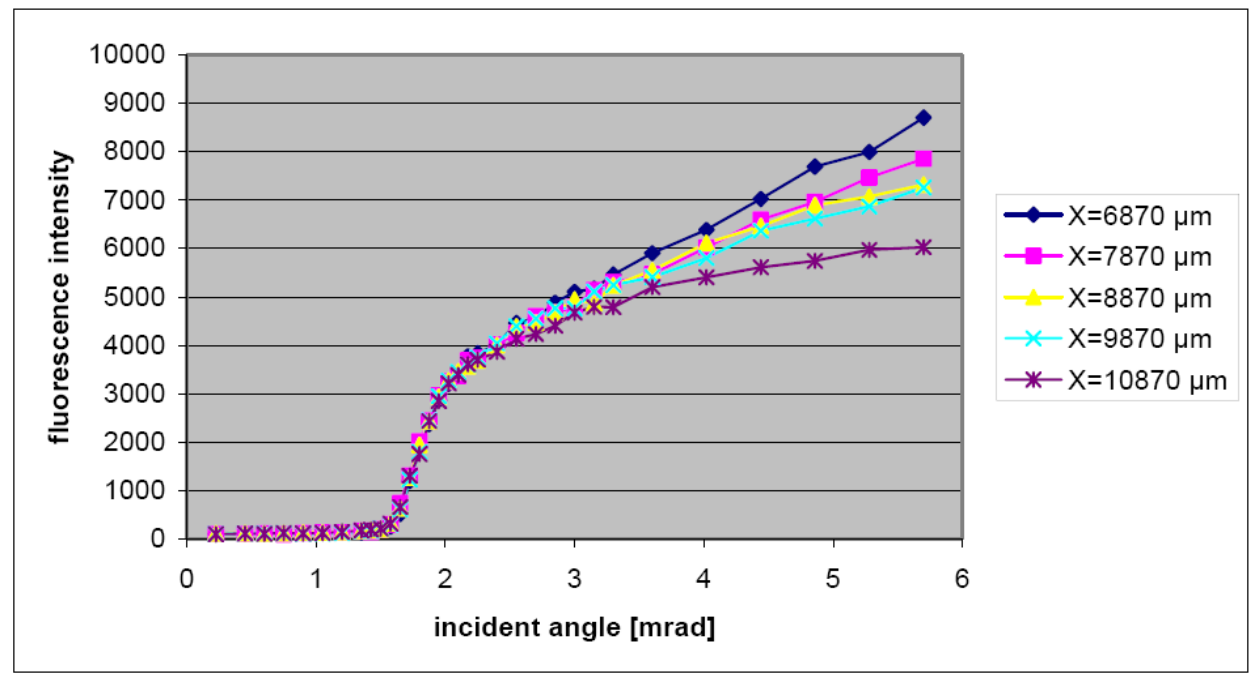

Figure 19: Influence of the Upstream/Downstream position $(X)$ on the measured Si curves. The pink curve represents the $\mathrm{X}$-value where measured and theoretical data fit best.

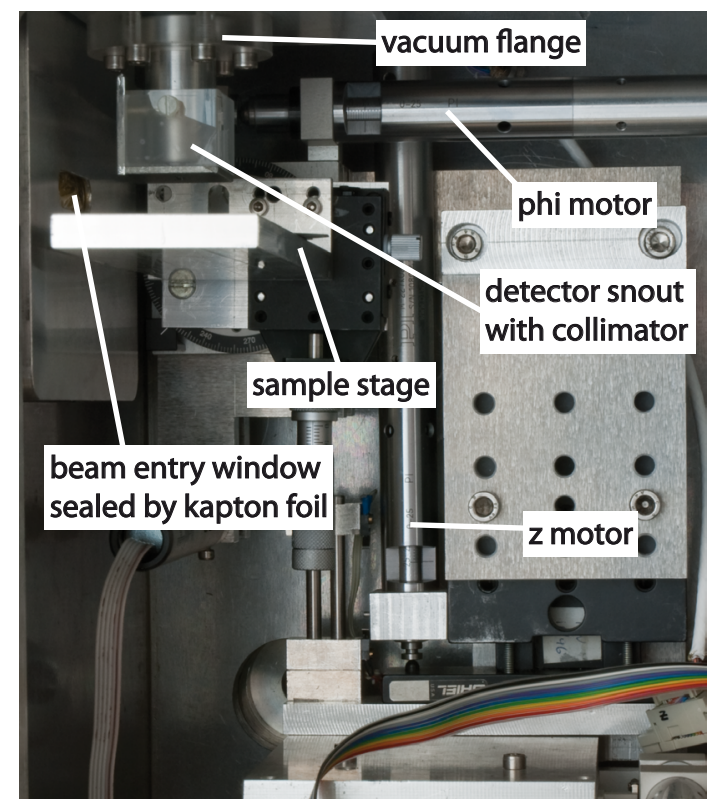

Figure 20: Interior view of the GIXRF spectrometer 


\subsection{XRF detector}

Another important part of thin-film analysis is the detector system, which has to chosen carefully as several features are required especially for thin-film analysis. To keep the angular divergence of the scan as low as possible a slit collimator in front of the detector window is preferable which has to be aligned parallel to the Y-axis. Its slit width should be chosen to prevent that fluorescence can enter the detector which is generated by the primary beam at lower/larger angles than adjusted - a case that always occurs due to the divergence of the exciting beam. Generally the used detector is a Silicon drift detector (SDD) and is mounted perpendicular and very close (distance collimator - sample $\sim 1 \mathrm{~mm}$ ) to the reflector surface to obtain a spectrum with a minimum background due to scattering from air. Another advantage of this small distance is, that the slit collimator covers a large solid angle in the plane defined by its aperture which enhances the detected fluorescence intensity. Finally the detector itself has to be capable of processing very high count rates (several kcps) as the fluorescence intensity of the substrate material (bulk intensity profile) increases drastically for angles larger than the critical angle of total reflection. This implies that the signal processing unit of the detector system has to be able to perform an accurate dead-time correction for a large working range (very low to very high countrates).

In the present design a silicon drift detector (SDD, Vortex, from Radiant) with an active area of $50 \mathrm{~mm}^{2}$ is used. The detector offers an energy resolution of $140 \mathrm{eV}$ with $12 \mu$ s shaping time at $5.9 \mathrm{keV}(\mathrm{Mn}-\mathrm{Ka}$ ) and a maximum processable count rate of $100 \mathrm{kcps}$. A custom-made collimator made of Molybdenum with a slit width of 3 millimeters is used. 


\section{Control Software}

A dedicated acquisition and control software was developed, in order to automate the necessary movement of motors and read-out of detectors during scans as much as possible. The software, written in C\# and C++/CLI, follows a modular approach, i.e. the devices, their parameters and the path to the corresponding dynamic link libraries are loaded from a configuration file, which is specified by the user after program startup. Thus it is easily possible to adapt it to different measurement setups or changing requirements. An overview of the devices will be given in section 4.1, while the structure of the configuration file will be discussed in section 4.2 .

It consists of a main window, which shows the actual spectra as well as the current status of the devices and allows the user to enter commands via a command line, and a scan window, which shows information on measured data during and after a scan. For further details see section 4.3.1 respectively 4.3.2.

Several common tasks like scan or count are implemented as builtin commands. A detailed reference of builtin commands and device commands can be found in appendix $\mathrm{A}$.

\subsection{Devices}

Devices are the main way to interact with the software as each builtin command is in principle also a device. Each device has, depending on its type, a list of properties and commands, which can be read, modified or executed by the user. The only common property for all devices is a name, which is used in the status display or to target it via command line. The name of loaded devices is specified in the configuration file, while the name of builtin devices is hardcoded. Common commands for loaded devices are: get, set and list to read or change settings; status; connect and disconnect.

\subsubsection{Motors}

The devices of type motor implement a special interface, which contains commands and properties for motor movement and status readout. These devices can contain code for physical devices, i.e. the code for the direct communication with the motor controller or device driver, or virtual devices, which execute combined or translated movements of other motor devices. The common commands for all motors are mva and mvr for absolute and relative movement. Common settings are: units (the display string for the motor units), factor (a conversion 
factor from hardware units to display units), max and min as software limits for the movement and position (the actual position in display units).

Code for the following controllers is currently implemented and tested:

- Compumotor CX

- Nanotec

- Physik Instrumente C-843

- Physik Instrumente C-863 via GCS2

It is also worth mentioning that the Physik Instrumente devices allow backlash compensation by performing additional automatic movement, which can be set via the 'backlash' setting (in motor units).

Additionally a virtual device was implemented, which calculates the necessary corrections to translate a linear, tangential movement to an angle.

\subsubsection{Detectors}

The detector interface defines commands, which have to be implemented by the device code for the acquisition and readout of spectrum data from a digital signal/pulse processor or multichannel analyzer. The common commands for all detector devices include count to start the acquisition and save to store a spectrum to disk, as well as commands to add (roiadd) or remove (roidel) rois. The net and gross sums of the channels within the roi will be shown in the status and will be available for display in the scan window.

The code for support of the following devices is currently implemented:

- Amptek DP5

- Ketek AXAS-M

- Radiant Vortex

\subsection{Configuration file}

The configuration file is a file in xml syntax, which contains the names and settings of all devices, which should be loaded. Listing 1 shows an example of one device section in this file, a complete listing of a configuration file can be found in appendix A.5.

Listing 1: Section in the configuration file for loading a motor.

$<$ add name $=" h "$ assemblyName $="$ AtiMotorPIGCS2 . d11"> $<$ settings $>$

$$
<\text { clear } />
$$




$$
\begin{aligned}
& <\text { add key="position" value ="54457.916000" } \\
& \text { /> } \\
& \text { <add key="units" value="um" /> } \\
& <\text { add key="factor" value }=" 1000 " \text { / > } \\
& <\text { add key="max" value }=" 94000 " /> \\
& <\text { add key }=" \min " \text { value }=" 20000 " \text { / > } \\
& <\text { add key="axisid" value }=" 1 " \text { / > } \\
& <\text { add key="stageid" value="M-410.CG" /> } \\
& <\text { add key }=" \text { controllerdesc" value }=" \text { PI C }-863 \\
& \text { Mercury SN 0125500361" /> } \\
& \text { <add key="settletime" value }=" 0.5 " \text { / > } \\
& \text { <add key="windowon" value }=" 1 " \text { /> } \\
& \text { <add key="windowoff" value }=" 4 " \text { /> } \\
& <\text { add key="backlash" value }=" 3 " \text { / > } \\
& </ \text { settings }> \\
& </ \text { add }>
\end{aligned}
$$

Each device entry must have an unique name and specify the assemblyName, i.e. the filename, of the dynamic link library, which contains the actual code for the communication with the motor controller. The settings of the device are specified in key-value pairs and depend on the actual device type.

\subsection{Graphical User Interface}

\subsubsection{Main Window}

After starting the software, the first window to open will be the main window (Figure 21). It is divided intro three parts: a tabcontrol containing a tab with a spectrum for each detector, a status display and a command line. The spectrum and status display will refresh automatically depending on the readout speed of the connected hardware. While a device is executing a command (listed as BUSY), the command line is locked. In order to interrupt execution, the user has to press Ctrl-C. This will issue a stop command to all connected motors and detectors. Using the up and down arrow keys in the command line will cycle through previously used commands.

A typical startup sequence is:

1. load a configuration file by entering its name in the command line

2. connect all devices at once by using connect or individually by <device> connect 


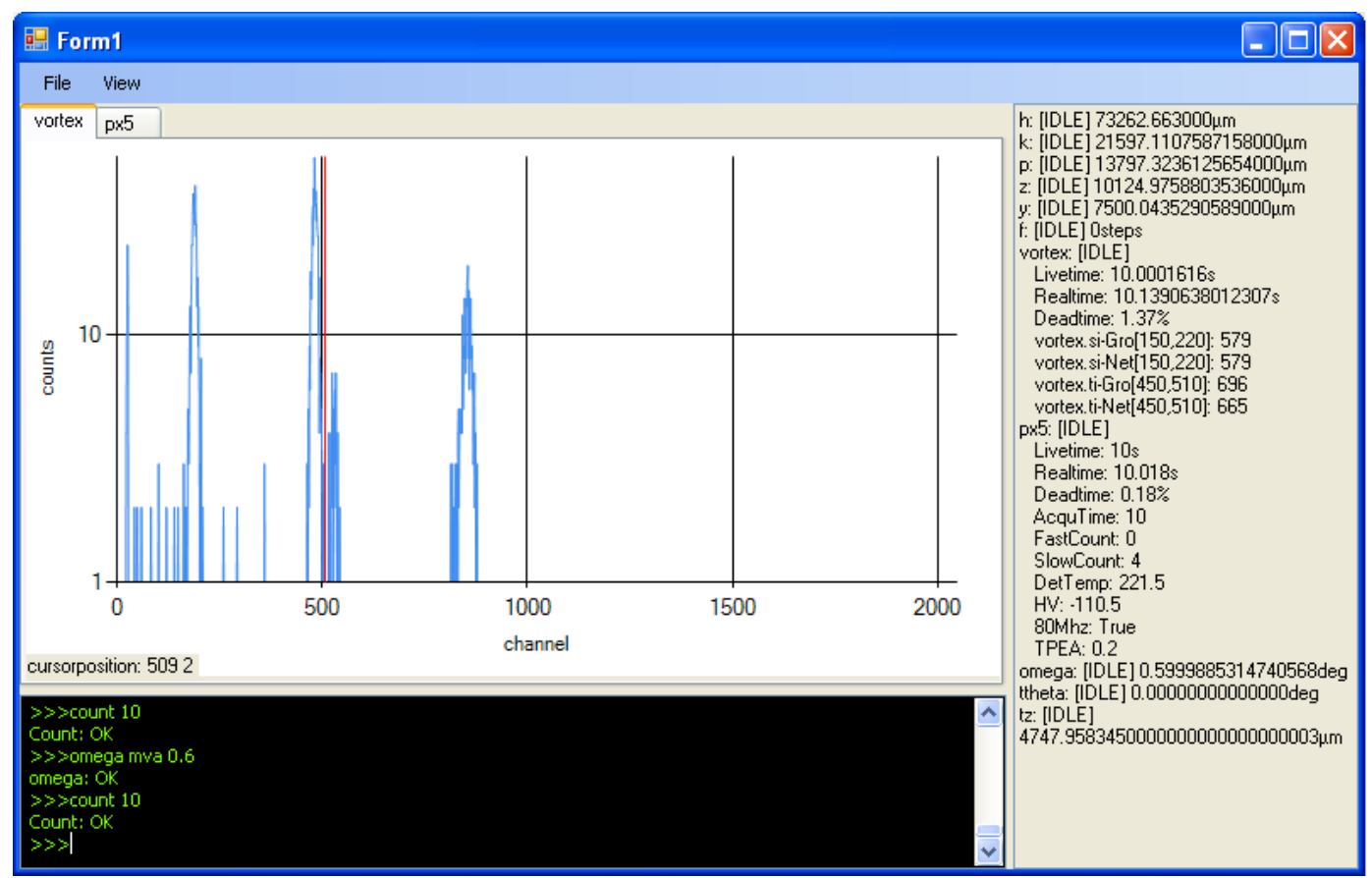

Figure 21: Main window of the GUI

3. use roiadd on a detector to set rois

\subsubsection{Scan Window}

The scan window shows a graph containing measurement data of the current scan and opens automatically when the first scan after program startup is started. As can be seen from Figure 22, which shows data from an angle (omega) scan, and Figure 23, which shows a scan of the $\mathrm{z}$ axis, the selected and displayed motor axis can be changed in the corresponding list box. The display of detector rois or other data in the graph can be enabled or disabled via checkboxes. 


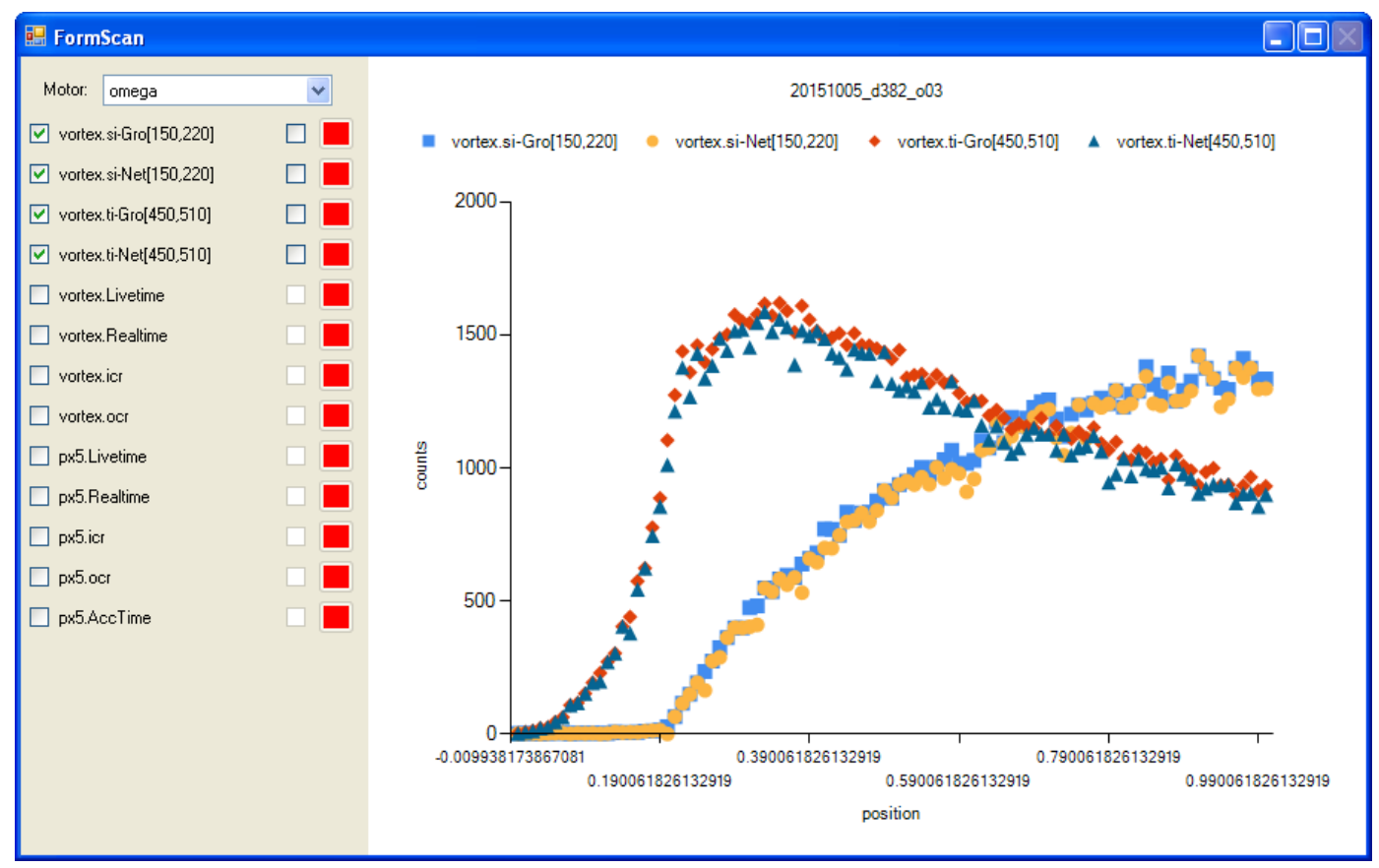

Figure 22: Scan window of the Graphical User Interface

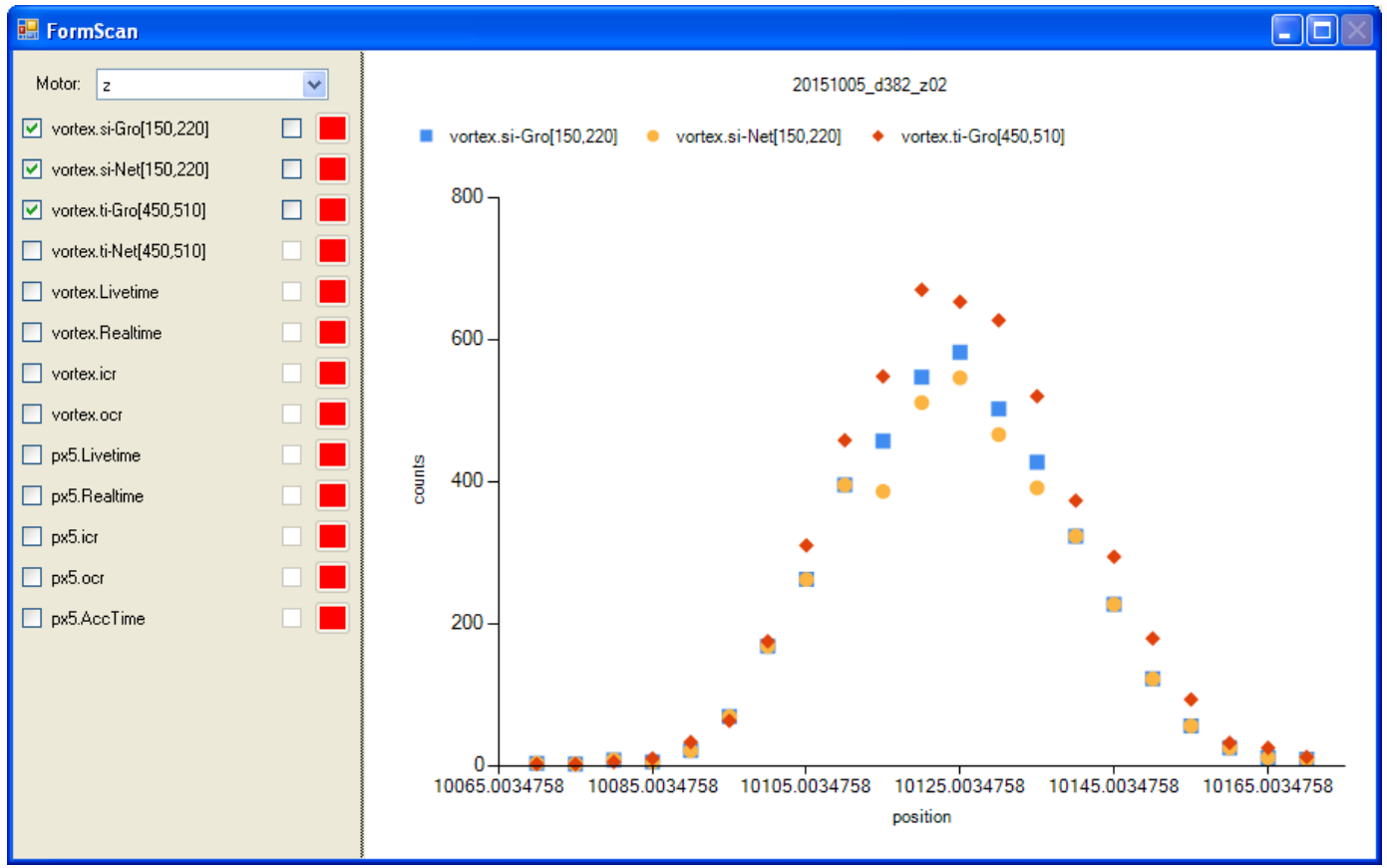

Figure 23: Scan window of the Graphical User Interface 


\section{Results and discussion}

All GIXRF measurements were performed using the prototype for GIXRF analysis, which was developed at the Atominstitut during participation in the European Integrated Activity of Excellence and Networking for Nano and MicroElectronics Analysis (short ANNA) of the European Commission Research Infrastructure Action under the FP6 "Structuring the European Research Area" Programme (project no. 26134). The samples were part of the same project. The results have been published in [10], [11] and [12].

The following parameters were used for the measurements:

- X-ray source: $3 \mathrm{~kW}$ Mo-tube LFF operated at $50 \mathrm{kV} / 40 \mathrm{~mA}$ and $50 \mathrm{kV} /$ $55 \mathrm{~mA}$

- Monochromator: Multilayer from a commercial TXRF wafer analyzer (Atomika 8010W)

- Detector: Vortex $50 \mathrm{~mm}^{2}$ SDD with provided Electronics

- Measurement (live) time: variable (20 - 120 seconds per point)

- Angular resolution of each scan: variable (23 - $115 \mu \mathrm{rad})$

- Measurements were carried out in air and in vacuum (typical $<1 \mathrm{mbar}$ ). The effect of the absorption for Si can be seen in Figure 24. Variable stepsize was used for the scans to gain a higher angular resolution at the critical angle of total reflection. Some mrad below and above the critical angle the angular increment was increased. The intensity differences in that region are getting smaller as the profile tends toward a constant value. For the sake of a reduced overall measuring time the angular resolution is decreased in these regions. Nevertheless they are important to measure, as these data are used for normalization. For all measurements the intensities recorded for the Si-K $\alpha$ line have been used for angle calibration. This is necessary as small differences between samples may result in a shift of the angle profile relative to the motorposition. Therefore the relation between motor position and absolute angle was recalibrated for each angular scan.

Several samples of Silicon wafers implanted with Arsenic with nominal implant dose from 1E14 to 1E15 and implant energy from 0.5 to $5 \mathrm{keV}$ were measured (for a detailed description see Table 1 in section 5.2). Aim of these measurements was to test the prototype and the acquisition software and compare the results with the findings of Secondary Ion Mass Spectrometry (SIMS) and Instrumental Neutron Activation Analysis (INAA).

For SIMS analysis As implanted samples were measured by D. Giubertoni of 


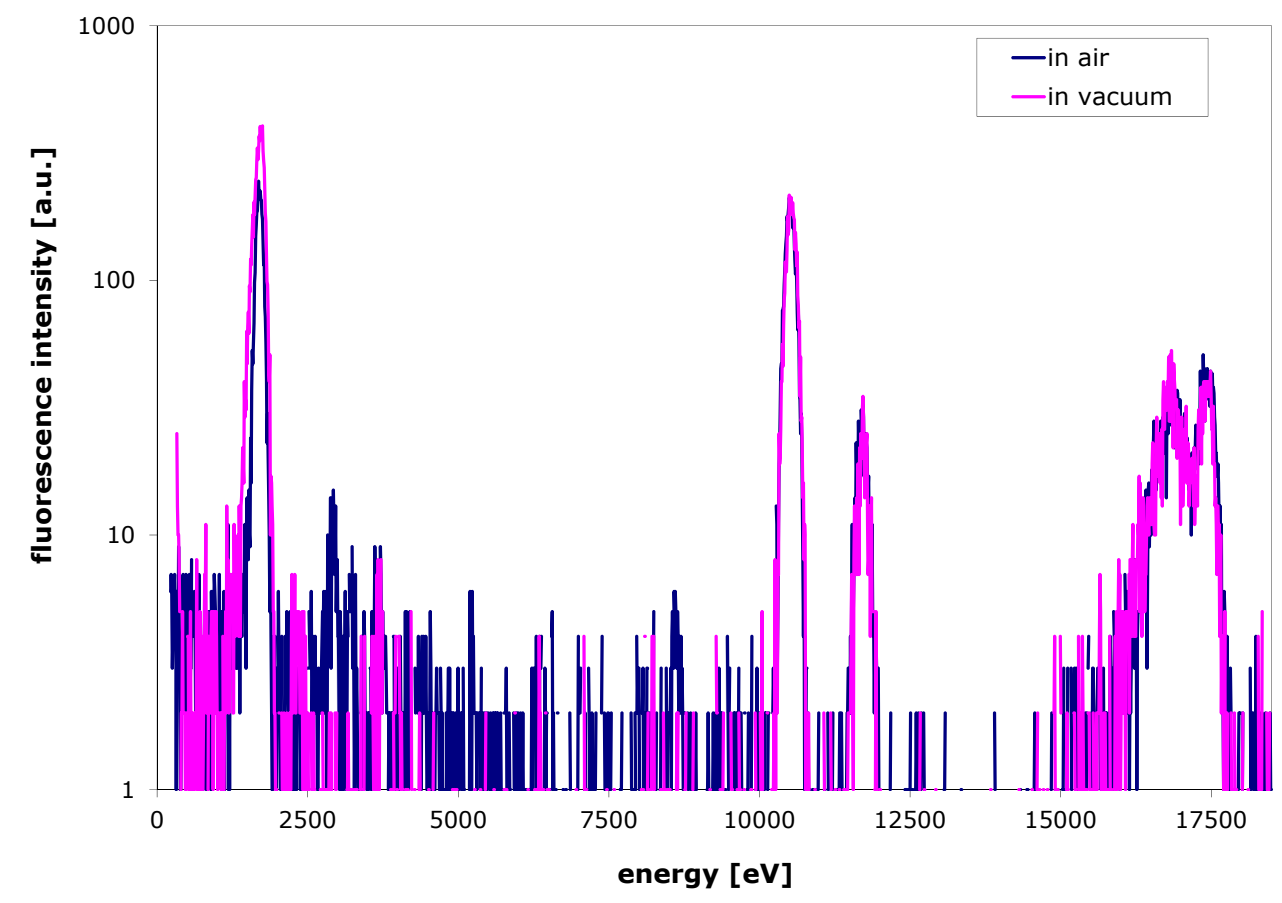

Figure 24: The same sample measured in air and in vacuum. The rest of the measurement parameters (angle, height, inspected area) were kept constant. Mo tube at $50 \mathrm{kV} / 55 \mathrm{~mA}, 20 \mathrm{~s}$ livetime.

Fondazione Bruno Kessler, Italy with a CAMECA Wf/SC-Ultra, magnetic sector secondary ion mass spectrometer, with a $\mathrm{Cs}^{+}$sputtering beam with impact energies of $500 \mathrm{keV}\left(\sim 45^{\circ}\right.$ incidence angle) and collecting ${ }^{28} \mathrm{Si}^{75} \mathrm{As}^{-}$and ${ }^{28} \mathrm{Si}_{2}{ }^{-}$ negative secondary ions. Sputtering time was converted to depth using a single constant sputtering rate as determined from the measurement of the final crater depth. ${ }^{28} \mathrm{Si}^{75} \mathrm{As}^{-}$secondary ion intensity was converted to As concentration using a relative sensitivity factor (RSF) determined from an As implant in Si of known dose. No corrections were applied to RSF in the initial transient width, surface $\mathrm{SiO}_{2}$ layer and its interface with $\mathrm{Si}$ substrate.

For the determination of As in bulk samples, INAA is a method of choice[31]. For the current problem, INAA measurements have been carried out by G. Steinhauser at the TRIGA Mark II reactor of the Atominstitut, Vienna University of Technology. Wafer pieces of about $3-4 \mathrm{~cm}^{2}$ were weighed into polyethylene vials, sealed and irradiated for $1200 \mathrm{~s}$ using the pneumatic sample transfer system. The neutron flux density in this position is approximately $3 \mathrm{E} 12 \mathrm{~cm}^{-2} \mathrm{~s}^{-1}$. After neutron irradiation, a cooling time of at least 60 minutes allowed the fast neutron activation products of silicon, ${ }^{28} \mathrm{Al}\left(\mathrm{T}_{1 / 2}=2.2414 \mathrm{~min}\right)$ and ${ }^{29} \mathrm{Al}\left(\mathrm{T}_{1 / 2}=6.56\right.$ $\mathrm{min}$ ) to decay almost completely and allowed an undisturbed measurement of 
the thermal neutron activation product ${ }^{76} \mathrm{As}\left(\mathrm{T}_{1 / 2}=1.0942 \mathrm{~d}\right)$. The measuring times were chosen independently for each sample, until the error due to counting statistics was $<5 \%$ rel. for the $559.1 \mathrm{keV}$ peak of ${ }^{76} \mathrm{As}$ in each spectrum. All samples were measured in a fixed position at a distance of approximately 10 $\mathrm{cm}$ beside the detector so that the slightest possible changes in the geometry of the sample next to the detector could be neglected. The $\gamma$-spectrometry was performed with a $222 \mathrm{~cm}^{3} \mathrm{HPGe}$-detector $(1.78 \mathrm{keV}$ resolution at the $1332 \mathrm{keV}$ ${ }^{60}$ Co peak; $48.2 \%$ relative efficiency), connected to a PC-based multi-channel analyzer with a preloaded filter and a Loss-Free Counting system. The area of the wafer pieces was calculated by derivation from the sample mass, Si density, and thickness. The total number of As atoms in the piece of wafer analyzed was determined by use of reference samples (semi-quantitative analysis).

For further testing of the GIXRF prototype and the acquisition software high- $\kappa$ (Hf) layer samples with layer thicknesses of 2 and $5 \mathrm{~nm}$ and varying composition were measured.

\subsection{Measuring protocol}

As the Si fluorescence signal is used to determine parameters necessary for quantification and dose determination special attention was drawn to the shape of the Si curve as a function of angle. After adjusting the height $(Z)$ of the sample, numerous scans were performed while varying the other motors of the sample stage (i.e. Theta, X, Y; one each time) systematically. Measured Si curves were fitted to the theoretical curves in order to check the quality of the experimental setup. Throughout these tests the influence of the different alignments (e.g. X-position, see section 3.2 and Figure 19) could be studied, which will be the foundation of the analysis protocol.

These measurements not only led to an improvement of the first GIXRF measuring chamber prototype, but also provided the basis for the GIXRF USJ measuring protocol. The gained insights allowed establishing an instruction guide which was carefully tested and improved during all analyses.

\subsubsection{Procedures I: System startup}

- Start cooling system. Prior to switching-on the generator system the cooling circuits have to be checked to prevent overheating.

- Switch on detector

- Follow generator warm-up procedure. Warm-up procedures specified by the manufacturers of the generator system have to be accomplished. 
- Check system status (adjusted / not adjusted). If the system is misaligned the adjustment procedure has to be performed with respect to the requirements of the planned experiments. Most of the steps of the adjustment procedure are routine actions (setting of tube, monochromator, slit system, detector height and adjustment of sample pivot).

- Start acquisition software.

- Setup of basic parameters. After the software startup the basic parameters for operation have to be set. This is done by loading a configuration file which enables communication between computer, detector and motors (see section 4.2). If some of the components are changed (e.g. the detector system is replaced) another configuration file has to be loaded containing the parameters for the new setup. This offers maximum flexibility of the system just requiring a small set of configuration files. In the next step the connection to motors and detector system has to be started (connect motor, connect mca) and a working directory should be defined (cd < directory $>$ ). All commands can easily be written to a batch file (standard text format, one line for each command) which can then be executed by a single command (batch $<$ filename $>$ ).

\subsubsection{Procedures II: Measurement}

- Open new protocol file(s). Each measurement series should be well documented. This is preferably done by keeping a log file of optional format.

- Insert sample and set region(s) of interest (ROI). After placing the sample on the sample holder (and potentially evacuation of the chamber) the region(s) of interest have to be set.

- Perform sample adjustment. The sample adjustment has to be performed to assure optimized alignment of the sample with respect to the incident beam and the detector. Only if all steps of the sample adjustment procedure are accomplished optimum performance of the system can be guaranteed. The whole process is illustrated in figure 25

- Start measurement(s). The measurement(s) should be performed using batch files allowing variable increments for the angular scan(s). The regions far above or below the critical angle of total reflection are needed to gain a good fit quality, but can be scanned with larger angular steps. At the critical angle on the other hand it is crucial to have a higher angular resolution. GIXRF spectra are measured and both the Si fluorescence intensity and the fluorescence intensity of the implant (e.g. As) are recorded for 
angles varying from 0 to 3 times the critical angle of total reflection.

\subsection{Arsenic Implants}

As the GIXRF measurement data represent the weighted integral of the depth profile up to a penetration depth of the X-rays, which depends on the incidence angle, and with the integral further modified by reflections on layers and standing wave patterns, the actual depth profile cannot be readily obtained. Based on an initial guess of the depth profile GIXRF data are calculated [4], compared to the measurement data and the guess modified until the calculation fits the measurement[30]. In the case of sufficiently smooth density changes (implants) or layers buried deeper in the substrate, the profile can not be resolved unambiguously[32]. Still in such circumstances GIXRF can be used for the verification of the quality of the profile given by other techniques (e.g. SIMS [9]) or combined with other methods like ion beam sputtering [33] or etching [34].

In the case of As implants a concentration profile obtained by SIMS was used as input and subsequently modified. GIXRF and SIMS are used as complementary techniques, as GIXRF is very sensitive in the first few nanometers, where SIMS is not accurate enough [35], while deeper in the substrate the situation is reversed.

The Si (bulk/substrate) curve is fitted to have an evaluation of three instrumental parameters:

1. the offset in the angle

2. the divergence of the beam

3. a flux/geometry factor (represented by a scale factor on the curve)

The total implanted dose can then be calculated according to [36] and [11]. Furthermore these parameters are used for processing and fitting of the Arsenic angular curve: The angle offset is used to correct the angular scale of the As intensity curve, the divergence value is used as initial estimate of the divergence in the As fit and the geometry factor is used as a scaling factor. In the calculation surface roughness has not been taken into account. In the fluorescence angular scan, a relevant roughness would be visible with an offset of the curve at small angles (higher fluorescence counts than expected at low angles). The SIMS profile is assumed to be correct in the depth and can be given some degrees of freedom in the surface layer. The modifications of the SIMS profile can be parameterized, and the parameters can be determined with a best fit procedure. In particular three parameters can be considered for the "correction" of the SIMS profile: (1) The depth of the correction, (2) the sputtering rate (modification/s- 


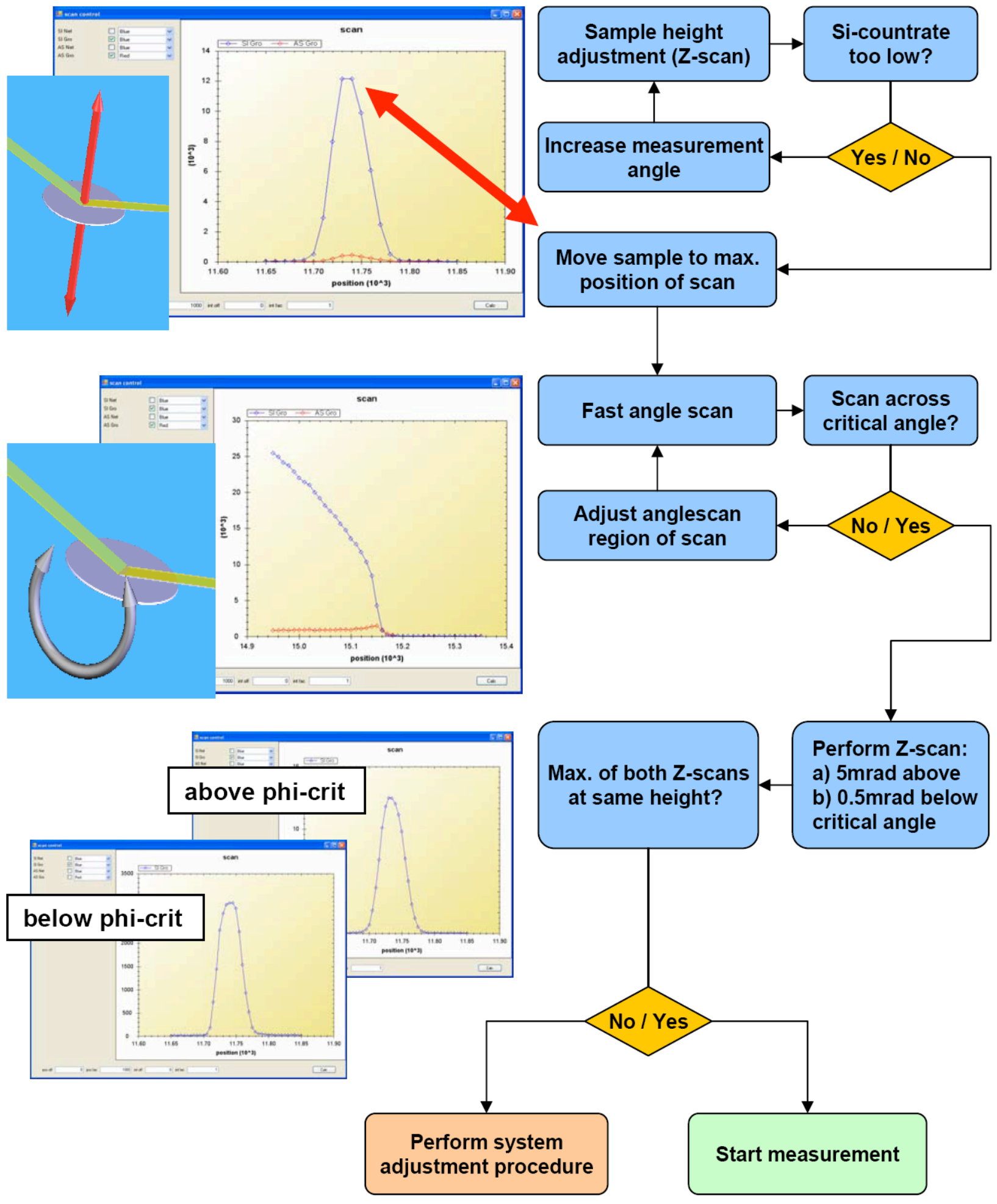

Figure 25: Illustration and flowchart of the sample adjustment procedure necessary to obtain optimum performance of the system for GIXRF measurements. 


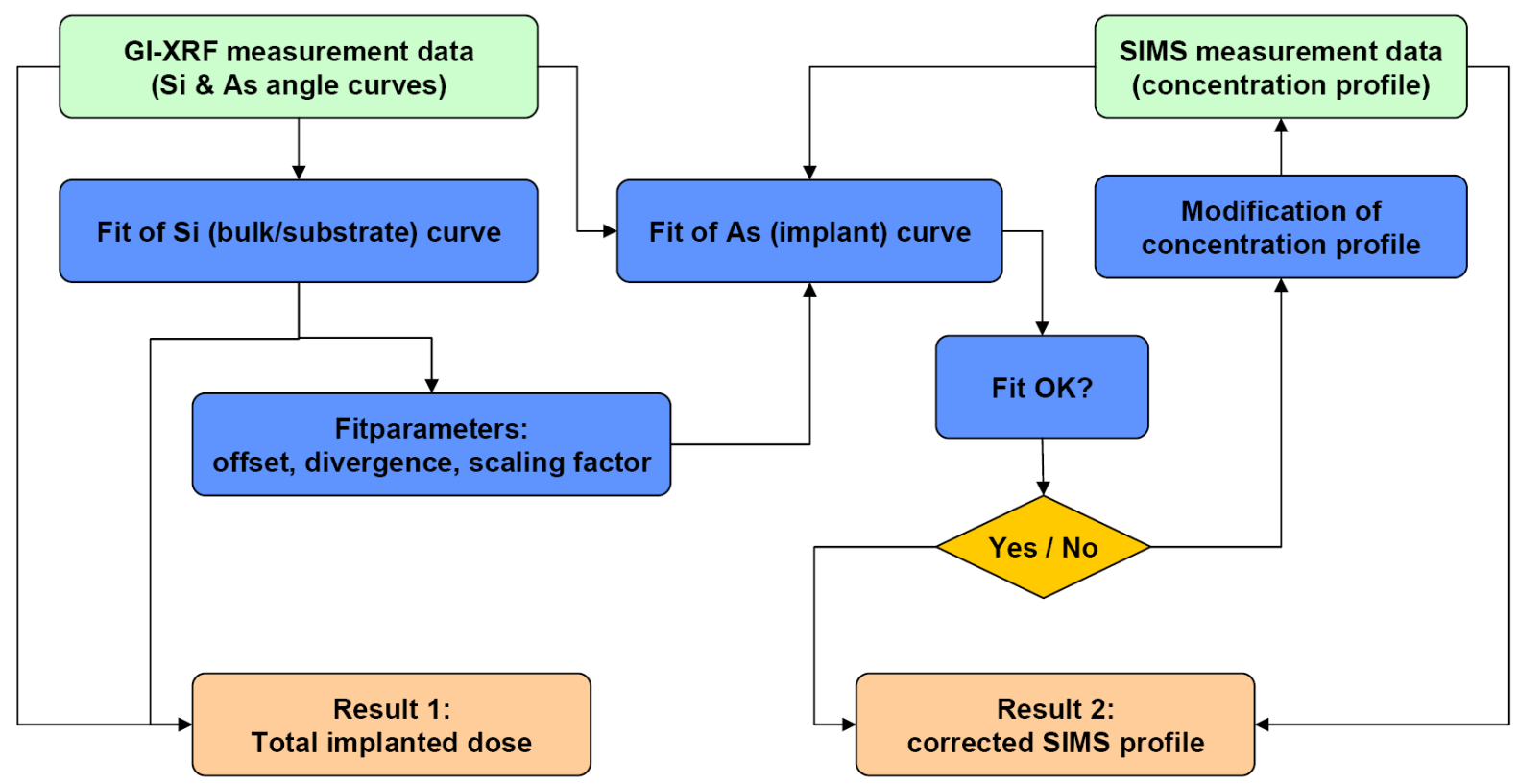

Figure 26: Flowchart of the evaluation process for GIXRF measurements in combination with SIMS profiles.

caling of the $\mathrm{X}$-axis) and (3) a relative sensitivity factor (modification/scaling of the Y-axis). An overview of the fitting procedure is shown in Figure 26.

Figures 27 and 28 show measured intensity profiles of Si and As respectively from sample Bes09 and a comparison with calculated curves. The X-ray tube was operated at $50 \mathrm{kV} / 40 \mathrm{~mA}$, acquisition time was $20 \mathrm{~s}$ livetime per point. The curves were calculated and modified for beam divergence using an algorithm and software developed by G. Pepponi [9]. By means of the iterative fitting algorithm described above the parameters matching the measured data were determined. The beam divergence was taken into account by convoluting the theoretical data with a Gaussian function. The FWHM value of the Gaussian function was varied until best agreement with the experimental data was reached. The final FWHM value determines the total divergence of the beam being the sum of angular and energy divergence. In the presented case a beam divergence of $0.3 \mathrm{mrad}$ in the calculation showed the best fit to the measurement. 


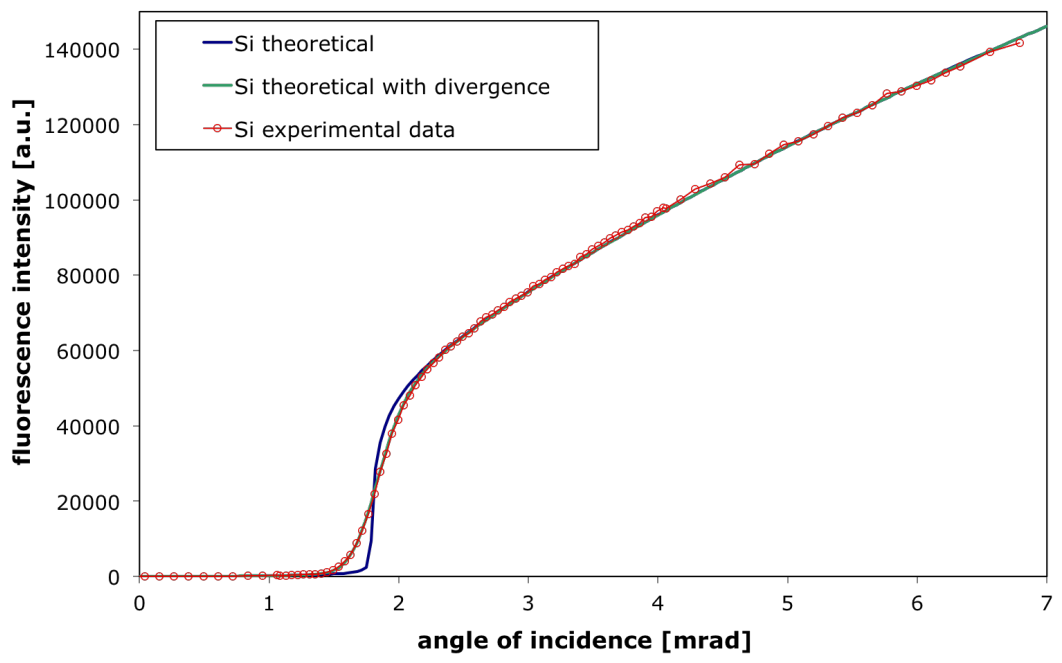

Figure 27: Si fluorescence data from a Si-wafer with As-implant (sample Bes09).

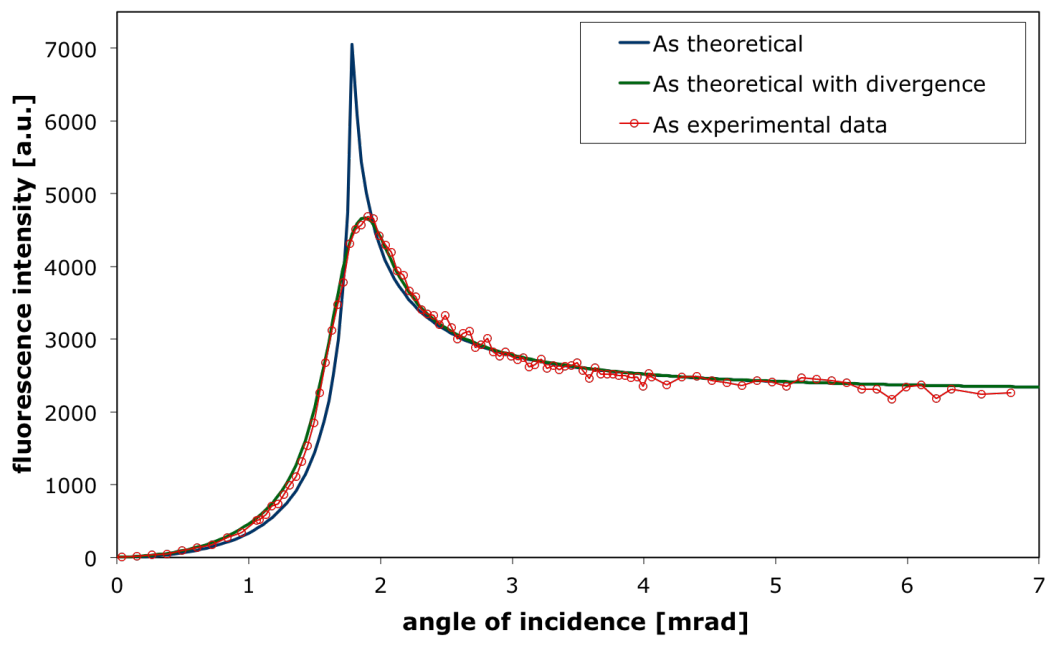

Figure 28: As fluorescence data from a Si-wafer with As-implant (sample Bes09). 
An overview of the measured samples with implantation energy and nominal implanted dose is given in Table 1. Bes09 and Bes18 are reference samples, which were used for quantification of the other samples.

\begin{tabular}{|c|c|c|c|c|}
\hline Sample Code & $\begin{array}{c}\text { Implanted } \\
\text { Species }\end{array}$ & $\begin{array}{c}\text { Implant Energy } \\
(\mathrm{keV})\end{array}$ & $\begin{array}{c}\text { Implant Dose } \\
\left(\text { atoms } / \mathrm{cm}^{2}\right)\end{array}$ & substrate \\
\hline \hline Standards & & & & \\
\hline Bes09 & As & 5 & $7.99 \mathrm{E}+14$ & $\mathrm{Si}$ \\
\hline Bes18 & As & 5 & $1.25 \mathrm{E}+14$ & $\mathrm{Si}$ \\
\hline ANNA As ref. & & & & $\mathrm{Si}$ \\
\hline As1 (Slot2) & As & 0.5 & $1.00 \mathrm{E}+15$ & $\mathrm{Si}$ \\
\hline As2 (Slot3) & As & 1 & $1.00 \mathrm{E}+15$ & $\mathrm{Si}$ \\
\hline As3 (Slot4) & As & 2 & $1.00 \mathrm{E}+15$ & $\mathrm{Si}$ \\
\hline As4 (Slot5) & As & 3 & $1.00 \mathrm{E}+15$ & $\mathrm{Si}$ \\
\hline As5 (Slot6) & As & 5 & $1.00 \mathrm{E}+15$ & $\mathrm{Si}$ \\
\hline As6 (Slot7) & As & 2 & $1.00 \mathrm{E}+14$ & $\mathrm{Si}$ \\
\hline As7 (Slot8) & As & 2 & $5.00 \mathrm{E}+14$ & $\mathrm{Si}$ \\
\hline As8 (Slot9) & As & 2 & $5.00 \mathrm{E}+15$ & \\
\hline
\end{tabular}

Table 1: Description with nominal implantation energy and dose of the measured implant samples. Bes09 and Bes18 were used as reference for the quantification of the other samples.

The dose values measured by GIXRF are compared with the results obtained with SIMS and INAA in Figure 29. The data of the GIXRF quantification shows good agreement with the nominal values and the other techniques (error $<10 \%$ for all measured samples as shown in Figure 30).

Figure 31 compares the experimental GIXRF data for sample As1 (1E15 atoms $/ \mathrm{cm}^{2}$ of As implanted at $3 \mathrm{keV}$ ) with the simulated GIXRF data for a SIMS $500 \mathrm{eV}$ profile, a TRIM simulation profile as well as those profiles changed by the fitting procedure. The profiles determined with SIMS using three different impact energies as well as a profile from a TRIM simulation of the implantation and the corresponding profiles changed by the GIXRF depth scale correction are shown in Figure 32. The GIXRF correction moves the SIMS profile into the depth, which is in agreement with the TRIM simulation. However the present correction seems to lead to a too deep junction depth. This is because GIXRF is very sensitive to the dopant concentration in the near surface region where SIMS is measuring a too high concentration of As atoms. 

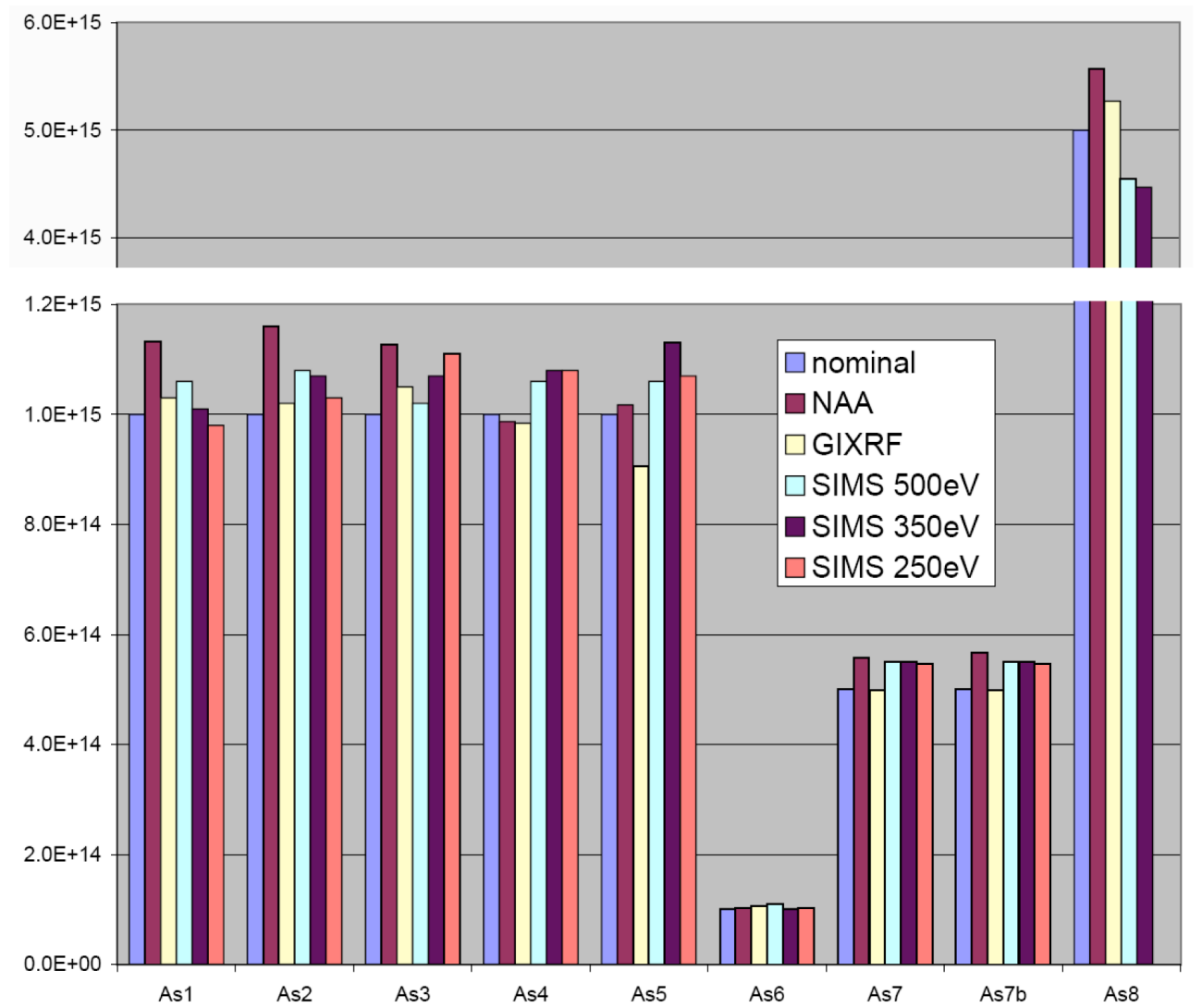

Figure 29: Comparison of the results of the total dose quantification with INAA, GIXRF and SIMS

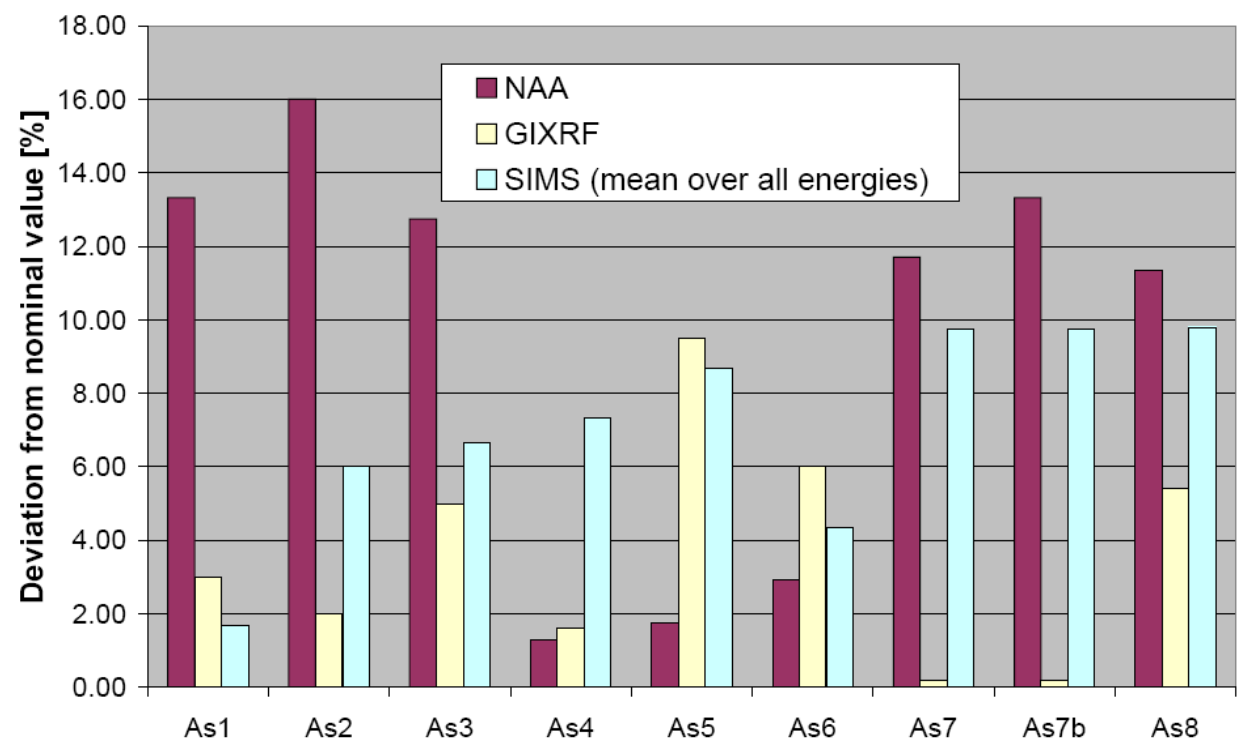

Figure 30: Absolute errors [\%] of the quantifications performed with NAA, GIXRF and SIMS. 


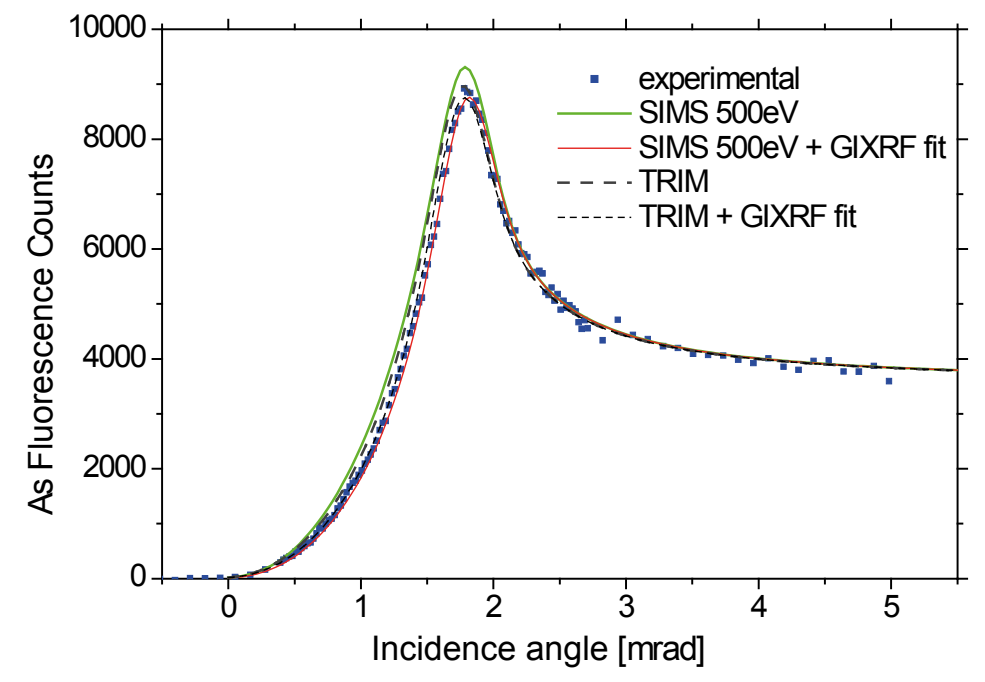

Figure 31: GIXRF experimental data and simulation of the fluorescence curve with the $0.5 \mathrm{keV}$ impact energy SIMS profile, the TRIM profile and those profiles changed by the GIXRF data fitting procedure. [11]

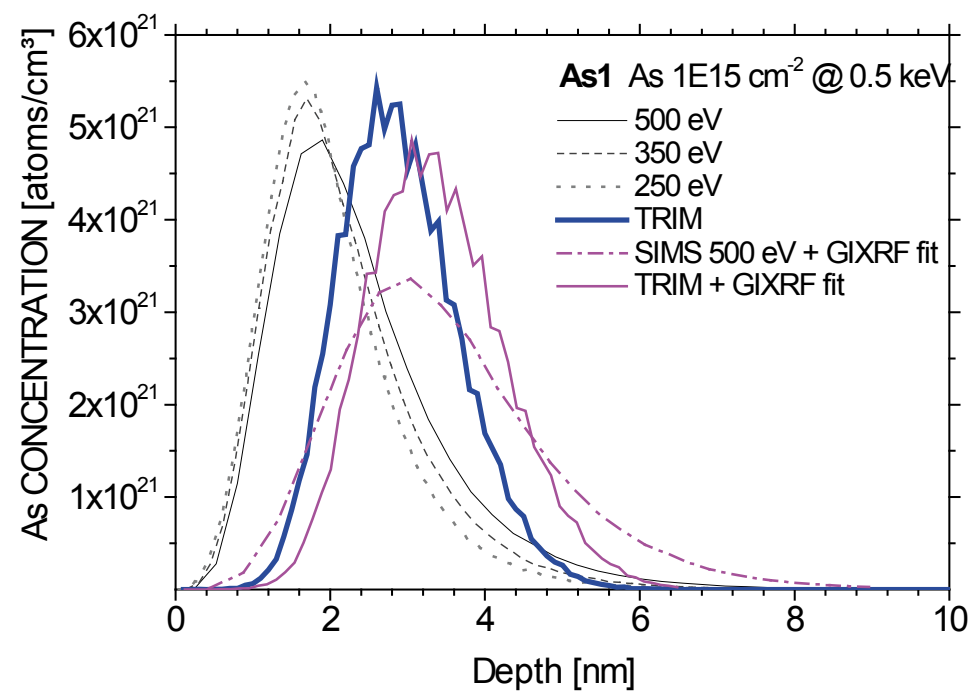

Figure 32: SIMS profile, TRIM simulated profile and GIXRF profile resulting from the change of the SIMS profile by means of the developed fitting procedure for the GIXRF data.[11] 


\subsection{Hafnium Oxide Layers}

Several samples of Silicon wafers coated with layers containing Hafnium in varying composition have been measured. An overview of the structure, nominal thickness and composititon of the samples is given in Table 2.

A qualitative comparison has been carried out, to evaluate the performance of the spectrometer. To compare the shapes of the measured profiles, the curves have been normalized. Each profile has been normalized according to:

$$
I=\frac{c p s-R 0}{R 1-R 0}
$$

where $c p s$ are the measured peak intensities of the Hf-L $\alpha$ line corrected for deadtime and normalized to counts per second, $R 0$ is the mean value of the first 5 data points of a scan and $R 1$ is the mean value of the last 5 data points of a scan. This normalization results in a profile where the region far below the critical angle is normalized to 0 whereas the angular region far beyond the critical angle is normalized to 1 .

From the normalized results shown in Figure 33 it is obvious that the shape of the $5 \mathrm{~nm}$ film profile is different from the ones for the $2 \mathrm{~nm}$ films. The maxima of the $2 \mathrm{~nm}$ profiles are located at the critical angle of Silicon (1.78 mrad for 17.5 $\mathrm{keV}$ excitation energy) whereas the $5 \mathrm{~nm}$ profile has no distinct maximum. This finding can be explained by reflections not only happening on the Si bulk layer, but also on the $\mathrm{HfO}_{2}$ layer. All these findings are in good agreement with theory and data from literature [29].

An estimation of layer thickness was performed by comparing and fitting the measured data to calculated curves. The results obtained with GIXRF were in good agreement with the thicknesses, which the ANNA cooperation partners determined by using other techniques like standard XRF, Medium Energy Ion Scattering (MEIS), Transmission electron microscopy (XTEM) and X-ray photo-

\begin{tabular}{|c|c|c|c|c|c|c|}
\hline Wafer & $1 \mathrm{~nm} \mathrm{SiO}_{2}$ & $\left.\mathrm{DPN}^{*}\right)$ & $5 \mathrm{~nm} \mathrm{HfO}_{2}$ & $2 \mathrm{~nm} \mathrm{HfO}_{2}$ & $\begin{array}{c}2 \mathrm{~nm} \mathrm{HfSiOx} \\
(60 \% \mathrm{Hf})\end{array}$ & $\left.\mathrm{DPN}^{*}\right)$ \\
\hline \hline D04 & $\mathrm{x}$ & & $\mathrm{x}$ & & & \\
\hline D05 & $\mathrm{x}$ & & & $\mathrm{x}$ & & \\
\hline D06 & $\mathrm{x}$ & & & $\mathrm{x}$ & & $\mathrm{x}$ \\
\hline D07 & $\mathrm{x}$ & & & & $\mathrm{x}$ & $\mathrm{x}$ \\
\hline D08 & $\mathrm{x}$ & & & & $\mathrm{x}$ & \\
\hline D09 & $\mathrm{x}$ & $\mathrm{x}$ & & & $\mathrm{x}$ & $\mathrm{x}$ \\
\hline
\end{tabular}

Table 2: Structure, nominal thickness and composition of the Hf oxide/silicate ultrathin layers deposited on Si wafers. ${ }^{*}$ DPN stands for decoupled plasma nitridation and post nitridation anneal (PNA) at $800^{\circ} \mathrm{C}$ to drive $\mathrm{N}$ into the layers 


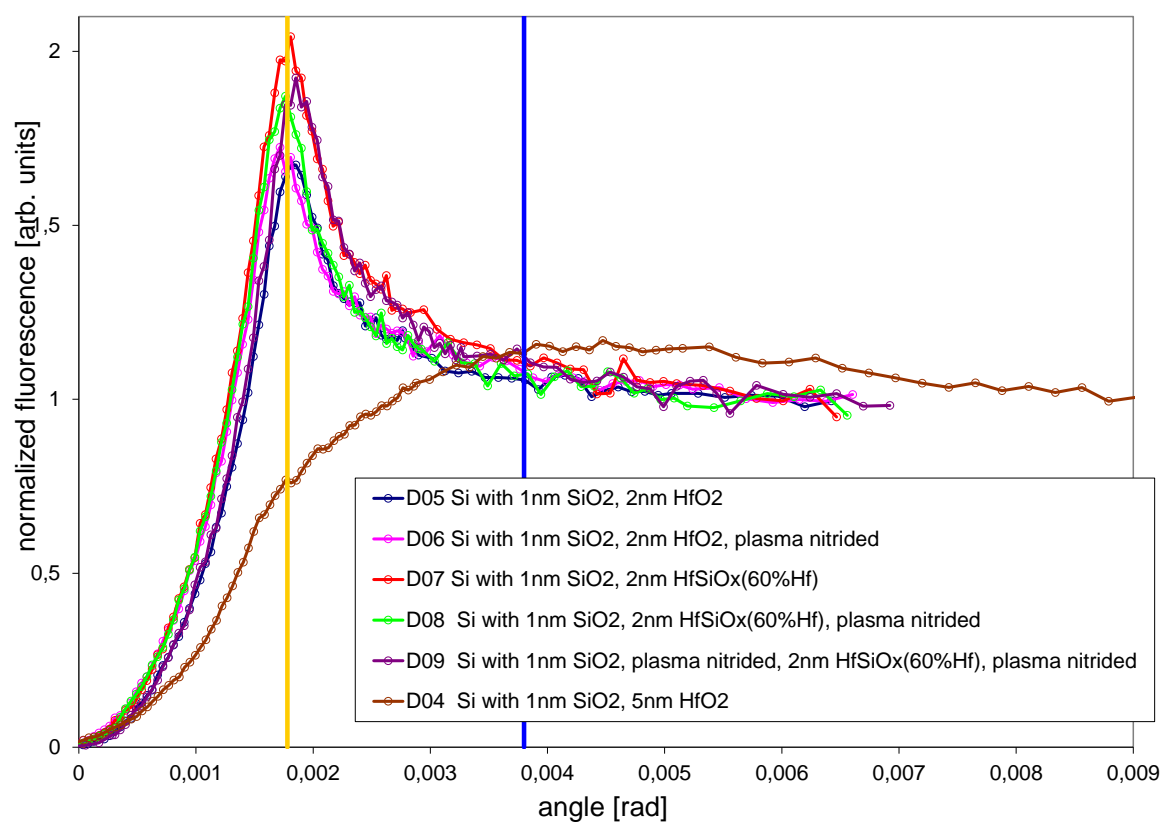

Figure 33: Measurement results of Hf-oxide nanofilm samples. All profiles have been normalized to their intensities recorded for angles far below / beyond the critical angle of total reflection. The vertical lines indicate the critical angle of total reflection of $\mathrm{Si}(1.78 \mathrm{mrad}$ at $17.5 \mathrm{keV})$ and $\mathrm{Hf}(3.8$ mrad at $17.5 \mathrm{keV})$.

electron spectroscopy (XPS). During the comparison of results within the ANNA consortium a discussion on layer densities was started and it was found that several techniques provide sensible and comparable layer thicknesses for different densities. Table 3 summarizes these results. The ambiguity concerning density and thickness is particular troublesome for samples, which contain layers of composite materials with unknown or imprecise values for density.

This circumstance instigated an investigation on the uniqueness of the results obtained by GIXRF and in fact it was found that the analysis yields ambiguous results (Figure 34 ). 


\begin{tabular}{|c|c|c|c|c|c|c|c|}
\hline \multirow{2}{*}{$\begin{array}{l}\text { Sample } \\
\text { code }\end{array}$} & \multirow{2}{*}{$\begin{array}{l}\text { Thickness } \\
\text { [nm] } \\
\text { (nominal) }\end{array}$} & \multirow{2}{*}{$\begin{array}{l}\text { Density } \\
{\left[\mathrm{g} / \mathrm{cm}^{3}\right]} \\
\text { (assumed) }\end{array}$} & \multicolumn{5}{|c|}{ Thickness [nm] (determined) } \\
\hline & & & XRF & MEIS & XTEM & XPS & GIXRF \\
\hline \multirow{2}{*}{ D04 } & \multirow{2}{*}{5} & 8.7 & 5.0 & 5.3 & & 5.2 & \\
\hline & & 9.7 & 4.5 & 4.8 & & 4.9 & 4.8 \\
\hline \multirow{2}{*}{ D05 } & \multirow{2}{*}{2} & 8.7 & 1.6 & 1.65 & \multirow{2}{*}{2.0} & 1.7 & \\
\hline & & 9.7 & 1.4 & 1.5 & & 1.6 & 1.6 \\
\hline \multirow{2}{*}{ D06 } & \multirow{2}{*}{2} & 8.7 & 1.4 & 1.75 & \multirow{2}{*}{1.8} & 1.6 & \\
\hline & & 9.7 & 1.3 & 1.6 & & 1.5 & 1.65 \\
\hline \multirow{2}{*}{ D07 } & \multirow{2}{*}{2} & 6.1 & 2.0 & 1.5 & \multirow{2}{*}{1.3} & 1.9 & 1.9 \\
\hline & & 6.7 & 1.8 & 1.4 & & & 1.65 \\
\hline \multirow{2}{*}{ D08 } & \multirow{2}{*}{2} & 6.1 & 2.1 & 1.5 & \multirow{2}{*}{1.4} & 1.9 & 1.9 \\
\hline & & 6.7 & 1.9 & 1.4 & & & \\
\hline \multirow{2}{*}{ D09 } & \multirow{2}{*}{2} & 6.1 & 1.4 & 1.6 & & 1.8 & 1.9 \\
\hline & & 6.7 & 1.3 & 1.5 & & & \\
\hline
\end{tabular}

Table 3: Compilation of results on $\mathrm{HfO}_{2}(\mathrm{D} 04, \mathrm{D} 05, \mathrm{D} 06)$ and $\mathrm{Hf}_{0.6} \mathrm{Si}_{0.4} \mathrm{O}_{\mathrm{x}}(\mathrm{D} 07, \mathrm{D} 08$, D09) layer thicknesses from the different techniques used within the ANNA project. An ambiguity concerning layer density and thickness was determined for several techniques.
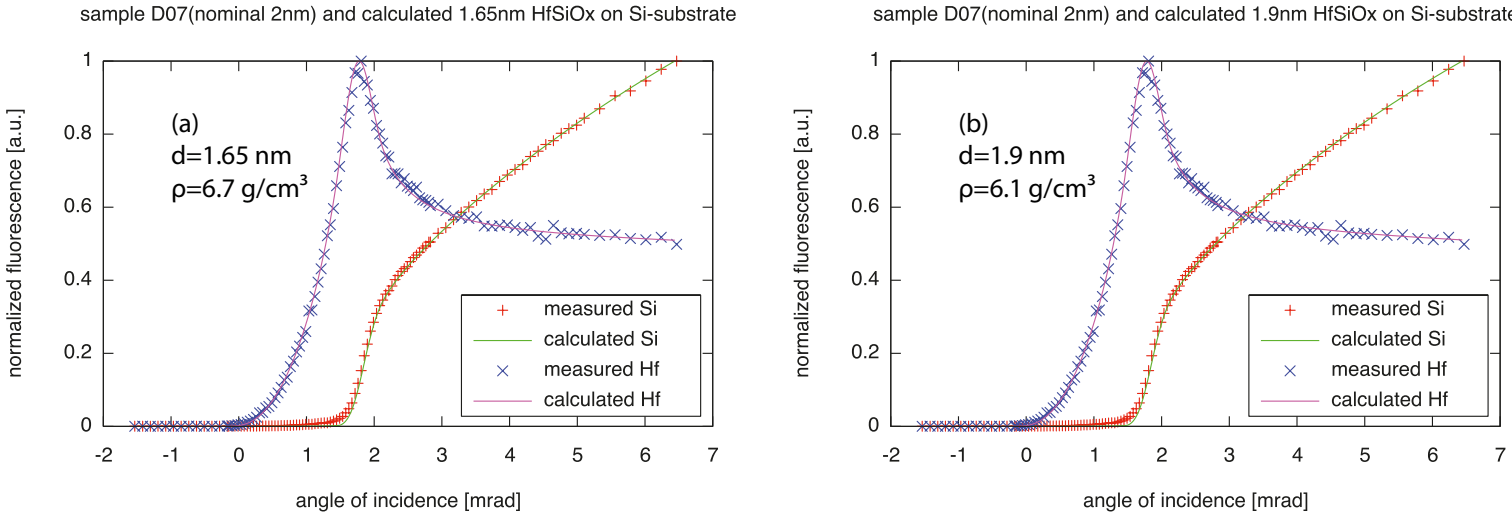

Figure 34: GIXRF measurement data fitted to calculated values. This comparison shows the ambiguity of GIXRF concerning density and thickness, a good fit can be found for a wide range of values. For the left side (a) a layer density of $6.7 \mathrm{~g} / \mathrm{cm}^{3}$ was used, while on the right (b) $6.1 \mathrm{~g} / \mathrm{cm}^{3}$ was used. This resulted in good fits for a layer thickness of $1.65 \mathrm{~nm}$ and $1.9 \mathrm{~nm}$ respectively. 


\section{Conclusions}

Summarizing the presented results it could be shown that the prototype GIXRF spectrometer developed for implant and nanofilm analysis works sufficiently well. The acquisition software provides a convenient and stable tool for angle scans, which can take several hours.

The results of implant measurements were in good agreement with theoretical predictions and the used fitting algorithm worked properly. Nevertheless the employed algorithm required input and restrictions on the profile shape from another technique (SIMS), as GIXRF alone does not provide enough information to determine an unique solution for the profile shape. The dose quantification showed good agreement with other techniques.

The measurements of thin film samples delivered curve shapes in accordance with literature data and confirmed the applicability of instrumentation and data acquisition software for thin-film analysis. But also in the case of layered samples it was found, that the obtained results contain an ambiguity concerning thickness and density. For all samples the determined values of layer thickness for a specific density were in good agreement with the other techniques, which were part of the ANNA project. Still this ambiguity is problematic especially for samples containing composite layers with limited or no knowledge on density.

As suggested by van den Hoogenhof and de Boer [7], a combination of GIXRF with X-ray reflectivity (XRR) measurements can provide more information on the sample and thereby reduce or eliminate this ambiguities. 


\section{References}

[1] D. De Boer, X-ray Standing Waves and the critical sample thickness for Total-reflection X-Ray Fluorescence analysis, Spectrochim. Acta 46 (1991) 1433.

[2] D. de Boer, W. van den Hoogenhof, TXRF of thin layers on and in solids, Adv. X-Ray Anal. 34 (1991) 35.

[3] D. De Boer, W. Van Den Hoogenhof, Total reflection X-ray fluorescence of single and multiple thin-layer samples, Spectrochimica Acta Part B: Atomic Spectroscopy 46 (10) (1991) 1323-1331. doi:10.1016/0584-8547(91) 80181-2

URL http://linkinghub.elsevier.com/retrieve/pii/0584854791801812

[4] D. de Boer, Glancing-incidence x-ray fluorescence of layered materials, Physical Review B 44 (2) (1991) 498-511. doi : 10.1103/PhysRevB.44.498. URL http://link .aps .org/doi/10.1103/PhysRevB.44.498

[5] D. de Boer, W. van den Hoogenhof, A. Leenaers, The profile of layered materials reflected by glancing-incidence X-ray analysis, Applied Physics A Solids and Surfaces 58 (3) (1994) 169-172. doi:10.1007/BF00324372. URL http://www . springerlink.com/index/10.1007/BF00324372

[6] D. de Boer, A. J. G. Leenaers, W. van den Hoogenhof, Glancing-incidence X-ray analysis of thin-layered materials: A review, X-Ray Spectrometry 24 (3) (1995) 91-102. doi : 10.1002/xrs.1300240304.

URL http://onlinelibrary.wiley.com/doi/10.1002/xrs.1300240304/ abstracthttp://doi.wiley.com/10.1002/xrs.1300240304

[7] W. Van Den Hoogenhof, D. De Boer, Glancing-incidence X-ray analysis, Spectrochimica Acta Part B: Atomic Spectroscopy 48 (2) (1993) 277-284. doi:10.1016/0584-8547(93) 80034-R,

URL http://linkinghub.elsevier.com/retrieve/pii/058485479380034R

[8] W. W. van den Hoogenhof, D. de Boer, Glancing incidence X-ray analysis: Forgotten or to be discovered?, Surface and Interface Analysis 22 (1-12) (1994) 572-575. doi :10.1002/sia.7402201121.

URL http://doi .wiley.com/10.1002/sia.7402201121

[9] G. Pepponi, C. Streli, P. Wobrauschek, N. Zoeger, K. Luening, P. Pianetta, D. Giubertoni, M. Barozzi, M. Bersani, Nondestructive dose determination 
and depth profiling of arsenic ultrashallow junctions with total reflection X-ray fluorescence analysis compared to dynamic secondary ion mass spectrometry, Spectrochimica Acta Part B: Atomic Spectroscopy 59 (8) (2004) 1243-1249. doi:10.1016/j .sab. 2004.04.014.

URL http://linkinghub.elsevier.com/retrieve/pii/ S0584854704001405

[10] D. Ingerle, F. Meirer, N. Zoeger, G. Pepponi, D. Giubertoni, G. Steinhauser, P. Wobrauschek, C. Streli, A new spectrometer for grazing incidence X-ray fluorescence for the characterization of Arsenic implants and Hf based high-k layers, Spectrochimica Acta Part B: Atomic Spectroscopy 65 (6) (2010) 429-433. doi:10.1016/j . sab.2010.02.015.

URL http://dx.doi.org/10.1016/j.sab.2010.02.015http:// linkinghub.elsevier.com/retrieve/pii/S0584854710000467

[11] G. Pepponi, D. Giubertoni, M. Bersani, F. Meirer, D. Ingerle, G. Steinhauser, C. Streli, P. Hoenicke, B. Beckhoff, Grazing incidence x-ray fluorescence and secondary ion mass spectrometry combined approach for the characterization of ultrashallow arsenic distribution in silicon, Journal of Vacuum Science \& Technology B: Microelectronics and Nanometer Structures 28 (1) (2010) C1C59. doi:10.1116/1.3292647. URL http://link.aip.org/link/JVTBD9/v28/i1/pC1C59/s1\{\&\}Agg=doi

[12] D. Giubertoni, G. Pepponi, B. Beckhoff, P. Hoenicke, F. Gennaro, F. Meirer, D. Ingerle, G. Steinhauser, M. Fried, P. Petrik, A. Parisini, M. A. Reading, C. Streli, J. van den Berg, M. Bersani, Multi-technique characterization of arsenic ultra shallow junctions in silicon within the ANNA consortium, AIP Conference Proceedings 1173 (2009) (2009) 45-49. doi:10.1063/1.3251258. URL http://www.scopus.com/inward/record.url?eid=2-s2. 0-70450228734\{\&\} partnerID=tZOtx3y1

[13] J. Als-Nielsen, D. McMorrow, Elements of Modern X-ray Physics, John Wiley \& Sons, Inc., Hoboken, NJ, USA, 2011. doi:10.1002/9781119998365 URL http://doi.wiley.com/10.1002/9781119998365

[14] H. Haken, H. C. Wolf, Atom- und Quantenphysik, Springer-Lehrbuch, Springer Berlin Heidelberg, Berlin, Heidelberg, 2004. doi:10.1007/ 978-3-642-18519-9.

URL http://link. springer.com/10.1007/978-3-642-18519-9

[15] R. Klockenkämper, A. von Bohlen (Eds.), Total-Reflection X-Ray Fluores- 
cence Analysis and Related Methods, John Wiley \& Sons, Inc., Hoboken, New Jersey, 2014. doi:10.1002/9781118985953.

URL http://doi .wiley.com/10.1002/9781118985953

[16] R. Jenkins, R. Gould, D. Gedcke, Quantitative X-Ray Spectrometry, 2nd Edition, Marcel Dekker, 1995.

[17] R. Woldseth, All You Ever Wanted To Know About X-ray Energy Spectrometry, Kevex, 1973.

[18] C. T. Chantler, Detailed tabulation of atomic form factors, photoelectric absorption and scattering cross section, and mass attenuation coefficients in the vicinity of absorption edges in the soft $X$-ray $(Z=30-36, Z=60-89$, $\mathrm{E}=0.1-10 \mathrm{keV})$, Journal of Synchrotron Radiation 8 (4) (2001) 1124-1124. doi:10.1107/S0909049501008305.

URL http://www.ncbi.nlm.nih.gov/pubmed/11486366http://scripts. iucr.org/cgi-bin/paper?S0909049501008305

[19] C. Whiston, X-ray Methods, Wiley, 1987.

[20] L. Strüder, P. Lechner, P. Leutenegger, Silicon drift detector - The key to new experiments, Naturwissenschaften 85 (11) (1998) 539-543. doi:10.1007/ s001140050545.

[21] E. Gatti, P. Rehak, Semiconductor drift chamber - An application of a novel charge transport scheme, Nuclear Instruments and Methods in Physics Research 225 (3) (1984) 608-614. doi:10.1016/0167-5087(84)90113-3.

URL http://linkinghub.elsevier.com/retrieve/pii/0167508784901133

[22] D. Attwood, Soft X-rays and Extreme Ultraviolet Radiation, Cambridge University Press, Cambridge, 1999. doi:10.1017/CB09781139164429.

URL http: //ebooks . cambridge.org/ref/id/CB09781139164429

[23] S. Ghose, B. Dev, X-ray standing wave and reflectometric characterization of multilayer structures, Physical Review B 63 (24) (2001) 1-11. doi:10.1103/ PhysRevB.63.245409.

URL http://link .aps.org/doi/10.1103/PhysRevB.63.245409

[24] P. Wobrauschek, Total reflection x-ray fluorescence analysis-a review, X-Ray Spectrometry 36 (5) (2007) 289-300. doi:10.1002/xrs.985.

URL http://doi.wiley.com/10.1002/xrs.985http://onlinelibrary. wiley.com/doi/10.1002/xrs.985/abstract 
[25] R. V. Grieken, A. Markowicz, Handbook of X-ray Spectrometry, Marcel Dekker, 2001.

URL http://scholar.google.com/scholar?hl=en $\{\&\} b t n G=\operatorname{Search}\{\&\} \mathrm{q}=$ intitle:Handbook+of +X-ray+Spectrometry\{\#\}0

[26] L. Fabry, S. Pahlke, Total reflection X-ray fluorescence analysis (TXRF), Surface and thin film analysis. Wiley, Weinheim.

URL http://scholar.google.at/scholar?q=L .+Fabry, +S.+Pahlke, +Total+reflection+X-ray+fluorescence+analysis+(TXRF) $\{\&\} \mathrm{hl}=$ de $\{\&\}$ as \{\}$_{-}$sdt $=0,5\{\&\}$ as $\left\{\_\right\}$ylo $=2002\{\&\}$ as $\left\{{ }_{2}\right\}$ yhi $=2002\{\#\} 3$

[27] P. Wobrauschek, P. Kregsamer, C. Streli, H. Aiginger, Recent developments and results in total reflection X-ray fluorescence Analysis, Adv. X-Ray Anal. 34 (1991) 1-12.

[28] K. N. Stoev, K. Sakurai, Review on grazing incidence X-ray spectrometry and reflectometry, Spectrochimica Acta Part B: Atomic Spectroscopy 54 (1) (1999) 41-82. doi:10.1016/S0584-8547 (98)00160-8.

URL http://www.sciencedirect.com/science/article/pii/ S0584854798001608http://linkinghub.elsevier.com/retrieve/pii/ S0584854798001608

[29] H. Schwenke, J. Knoth, U. Weisbrod, Current work on total reflection x-ray fluorescence spectrometry at the GKSS research centre, X-Ray Spectrometry 20 (6) (1991) 277-281. doi:10.1002/xrs .1300200606.

URL http://onlinelibrary.wiley.com/doi/10.1002/xrs.1300200606/ abstracthttp://doi.wiley.com/10.1002/xrs.1300200606

[30] U. Weisbrod, R. Gutschke, J. Knoth, H. Schwenke, Total Reflection X-ray Fluorescence Spectrometry for Quantitative Surface and Layer Analysis, Applied Physics A: Materials Science \& Processing 53 (5) (1991) 449-456. URL http: //www . springerlink.com/index/X2345M48R67417P8.pdf

[31] G. Steinhauser, W. Adlassnig, T. Lendl, M. Peroutka, M. Weidinger, I. K. Lichtscheidl, M. Bichler, Metalloid Contaminated Microhabitats and their Biodiversity at a Former Antimony Mining Site in Schlaining, Austria, Open Environmental Sciences 3 (1) (2009) 26-41. doi:10.2174/ 1876325100903010026 .

[32] H. Schwenke, J. Knoth, Depth Profiling in Surfaces Using Total Reflection X-Ray Fluorescence., Analytical Sciences 11 (3) (1995) 533-537. doi : $10.2116 /$ analsci.11.533. 
URL http://joi.jlc.jst.go.jp/JST.Journalarchive/analsci1985/11. 533? from=CrossRef

[33] H. Schwenke, J. Knoth, G. Wiener, Depth profiling using total reflection X-ray fluorescence spectrometry alone and in combination with ion beam sputtering, Acta Part B: Atomic.

URL http://www.sciencedirect.com/science/article/pii/ S0584854796016473

[34] R. Klockenkämper, A. von Bohlen, A new method for depth-profiling of shallow layers in silicon wafers by repeated chemical etching and total-reflection X-ray fluorescence analysis, Spectrochimica Acta Part B:

Atomic.

URL

http://www.sciencedirect.com/science/article/pii/ S058485479900066X

[35] A. Parisini, V. Morandi, S. Solmi, P. G. Merli, D. Giubertoni, M. Bersani, J. a. van den Berg, Quantitative determination of the dopant distribution in Si ultrashallow junctions by tilted sample annular dark field scanning transmission electron microscopy, Applied Physics Letters 92 (26) (2008) 261907. doi:10.1063/1.2951896.

URL http://scitation.aip.org/content/aip/journal/apl/92/26/10. $1063 / 1.2951896$

[36] P. Kregsamer, Fundamentals of total reflection X-ray fluorescence, Spectrochimica Acta Part B: Atomic Spectroscopy 46 (10) (1991) 1332-1340. doi:10.1016/0584-8547(91)80182-3.

URL http://www.sciencedirect.com/science/article/pii/ 0584854791801823http://linkinghub.elsevier.com/retrieve/pii/ 0584854791801823 


\section{A Software Reference}

\section{A.1 Builtin Commands}

\section{A.1.1 batch}

Executes commands, which are stored in a plain text file, line-by-line.

batch $<$ filename $>$

\section{A.1.2 cd}

Changes the current directory, i.e. the directory where scans and spectra are saved.

cd $<$ directory $>$

\section{A.1.3 connect}

Executes the connect command on a list of devices. If no device is specified, all devices are targeted.

connect $[<$ device $1>][<$ device $2>] \ldots$

\section{A.1.4 count}

Executes the count command on all detectors.

count $<$ seconds $>$

\section{A.1.5 disconnect}

Executes the disconnect command on a list of devices. If no device is specified, all devices are targeted.

disconnect $[<$ device $1>][<$ device $2>] \ldots$

\section{A.1.6 mca}

Sends a command to all detectors.

mca $<$ command $>$

\section{A.1.7 motor}

Sends a command to all motors.

motor $<$ command $>$ 


\section{A.1.8 mva}

Execute an absolute move on one or several motors.

mva $<$ motor $1><$ dest $1>[<$ motor $2><$ dest $2>] \ldots$

\section{A.1.9 mvr}

Execute a relative move on one or several motors.

mvr $<$ motor $1><$ dest $1>[<$ motor $2><$ dest $2>] \ldots$

\section{A.1.10 pwd}

Returns the current directory.

pwd

\section{A.1.11 save}

Saves the current spectra of all detectors to the files $<$ prefix $>_{\_}<$detectorname $>$.spe. save $<$ prefix $>$

\section{A.1.12 scan}

Executes a scan at $<$ intervals $>+1$ points with a measurement time of $<$ time $>$ seconds at every point by using one or several motors. A subdirectory <scanname $>$ is created in the current directory. All spectra and an summary text file are stored in this subdirectory. If the directory exists and the last scan had the same name, the data are appended to the existing data, otherwise an error is thrown. The motors are moved simultaneously. Useful e.g. for a $\theta-2 \theta$ scan, for an area scan use scan $2 \mathrm{~d}$.

scan $<$ scanname $><$ motor $1><$ from $1><$ to $1>[<$ motor $2><$ from $2><$ to $2>] \ldots$ $<$ intervals $><$ time $>$

\section{A.1.13 scan2d}

Executes an area scan at $<$ intervals $>+1$ points with a measurement time of $<$ time $>$ seconds at every point by using two motors. A subdirectory <scanname $>$ is created in the current directory. All spectra and an summary text file are stored in this subdirectory. If the directory exists and the last scan had the same name, the data are appended to the existing data, otherwise an error is thrown.

scan2d $<$ scanname $><$ motor $1><$ from $1><$ to $1><$ motor $2><$ from $2><$ to $2><$ intervals $><$ time $>$ 


\section{A.1.14 sleep}

Pauses the execution for $<$ n $>$ seconds, e.g. in a batch file.

sleep $<\mathrm{n}>$

\section{A.1.15 status}

Returns the status of all devices.

status

\section{A.2 Device Commands}

\section{A.2.1 connect}

Tries to establish communication with the hardware device and initialize it. $<$ device $>$ connect

\section{A.2.2 disconnect}

Terminates communication with the hardware device.

$<$ device $>$ disconnect

\section{A.2.3 get}

Reads a setting from the device.

$<$ device $>$ get $<$ setting $>$

\section{A.2.4 list}

Reads and lists all settings of the device.

$<$ device $>$ list

\section{A.2.5 set}

Sets a value on the device.

$<$ device $>$ set $<$ setting $><$ value $>$

\section{A.2.6 status}

Reads and displays the device status.

$<$ device $>$ status 


\section{A.3 Motors}

\section{A.3.1 mva}

Execute an absolute move.

$<$ motor $>$ mva $<$ dest $>$

\section{A.3.2 mvr}

Execute a relative move.

$<$ motor $>$ mvr $<$ dest $>$

\section{A.4 Detectors}

\section{A.4.1 count}

Initiates spectrum acquisition for $<$ seconds $>$.

$<$ detector $>$ count $<$ seconds $>$

\section{A.4.2 roiadd}

Adds a roi for display in the status display and in the scan windows.

$<$ detector $>$ roiadd $<$ name $><$ from $><$ to $>$

\section{A.4.3 roidel}

Removes a previously defined roi.

$<$ detector $>$ roidel $<$ name $>$

\section{A.4.4 save}

Stores the actual spectrum to disk. Two files are created: an AXIL compatible spe-file and a mca-file.

$<$ detector $>$ save $<$ filename $>$

\section{A.5 Configuration file}

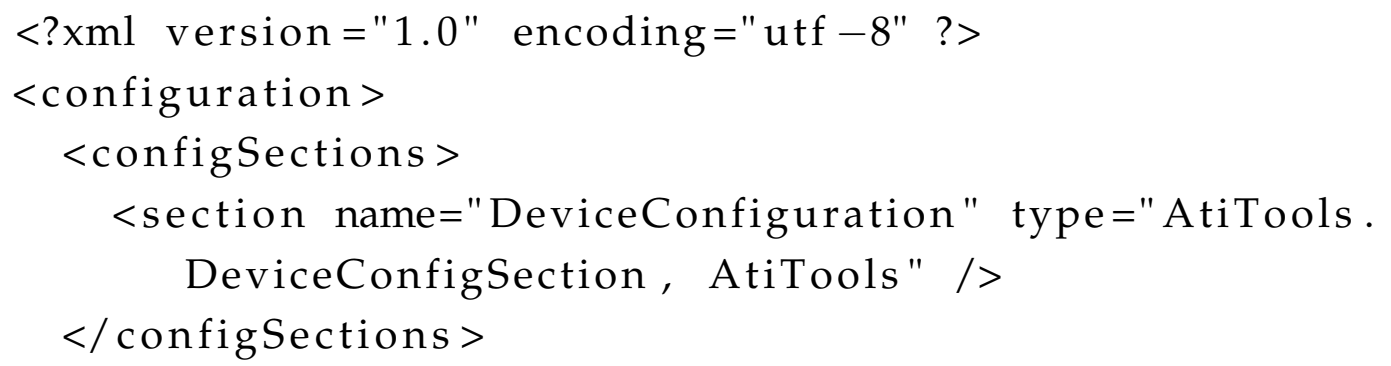




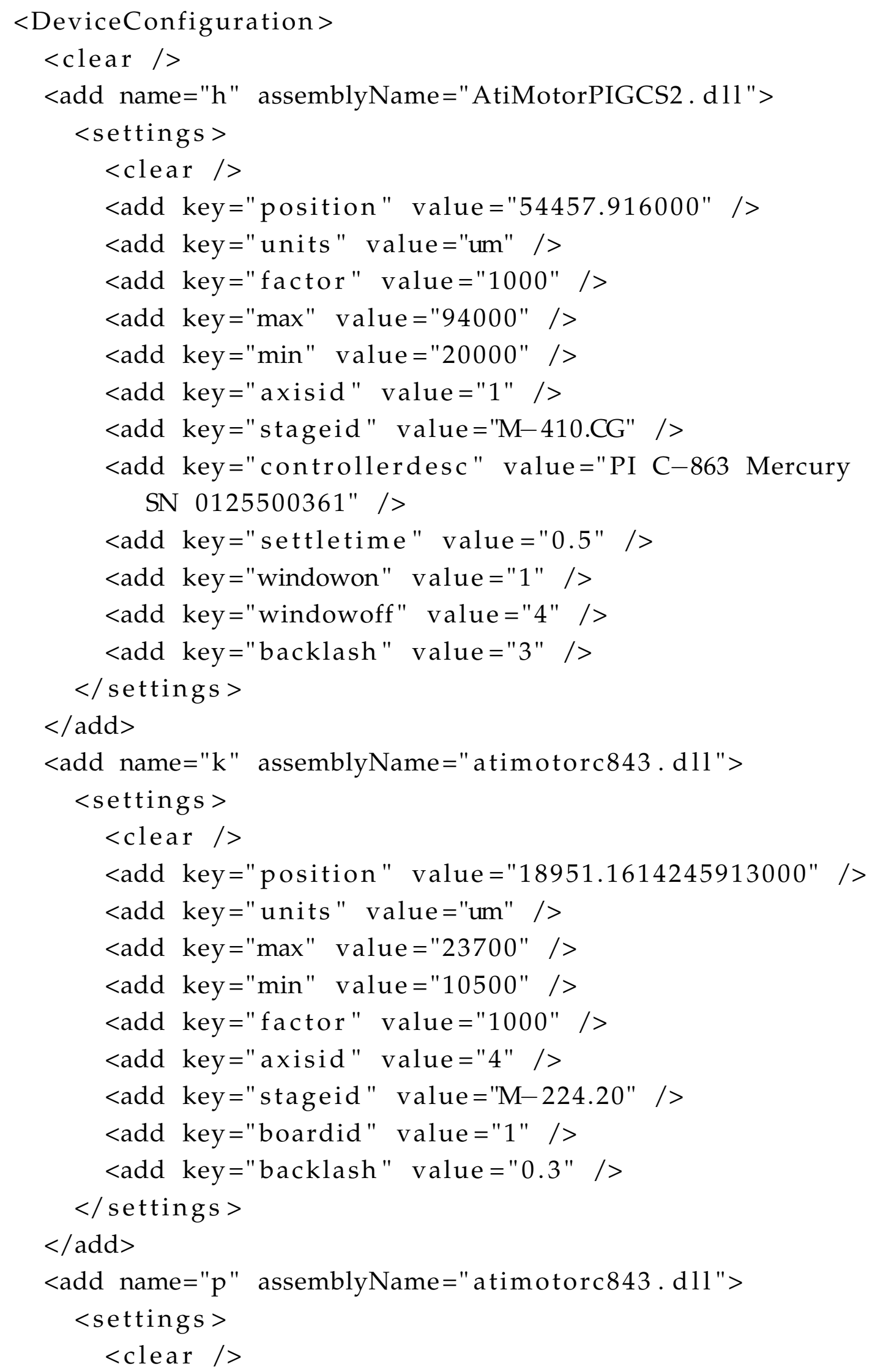




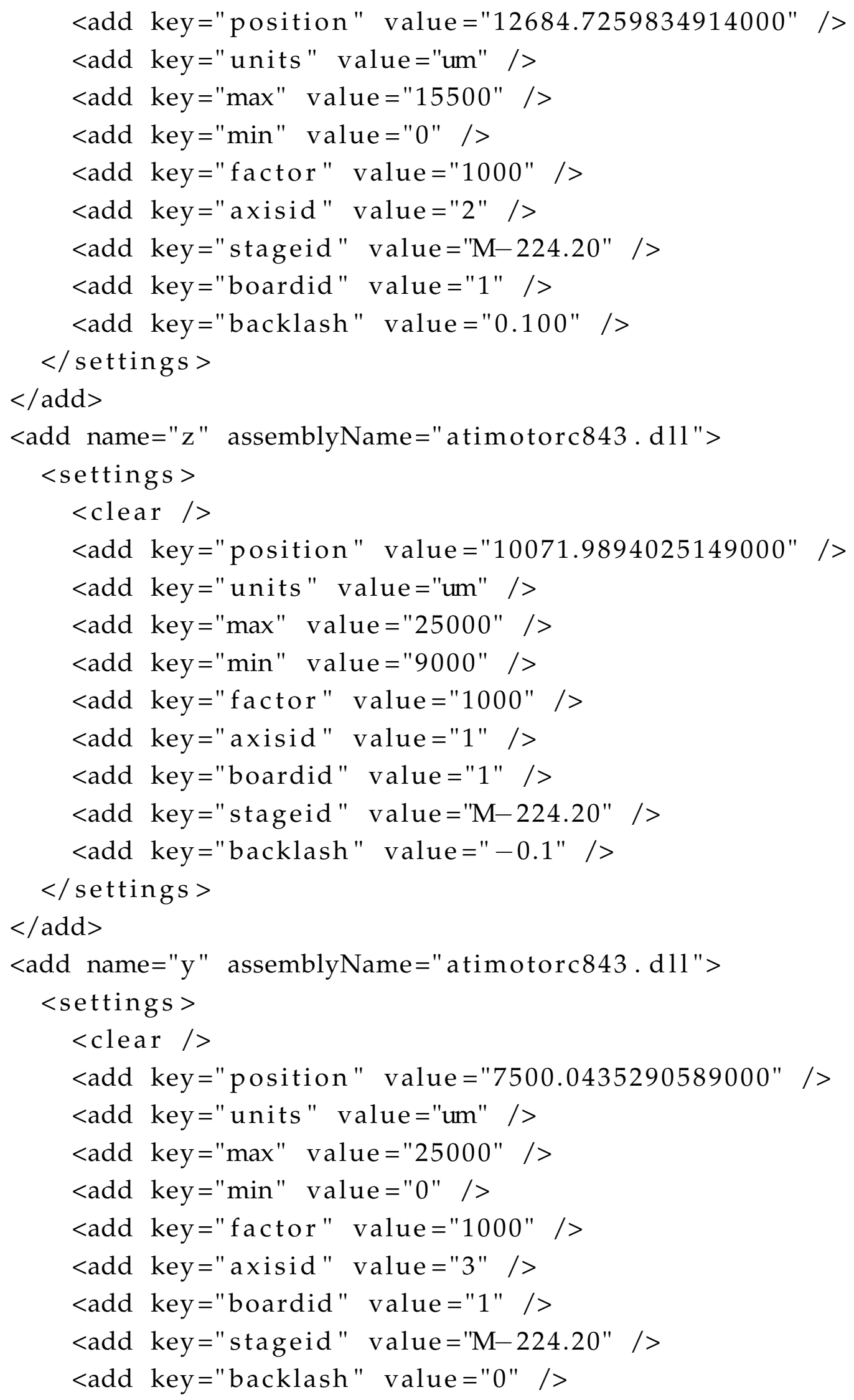




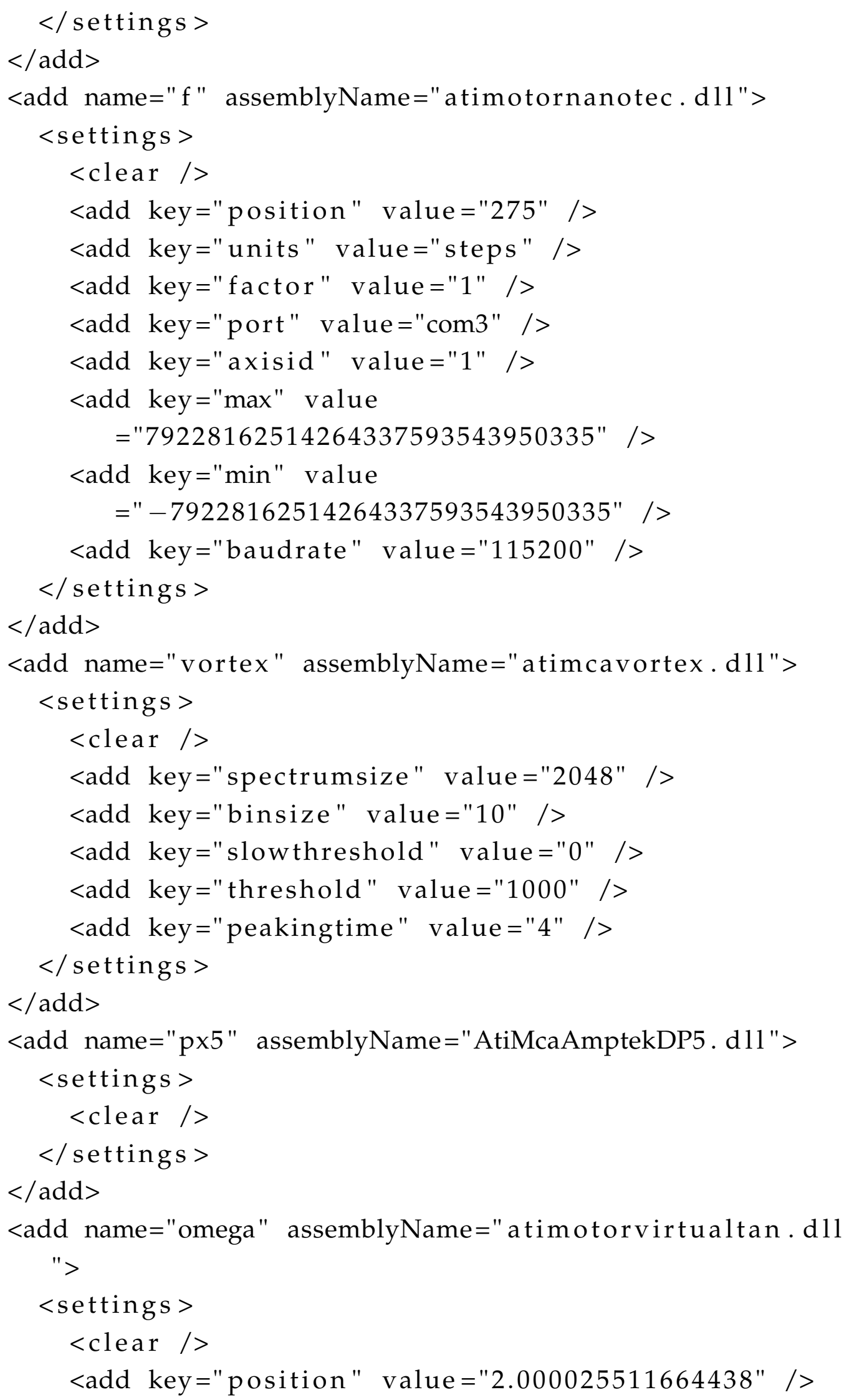




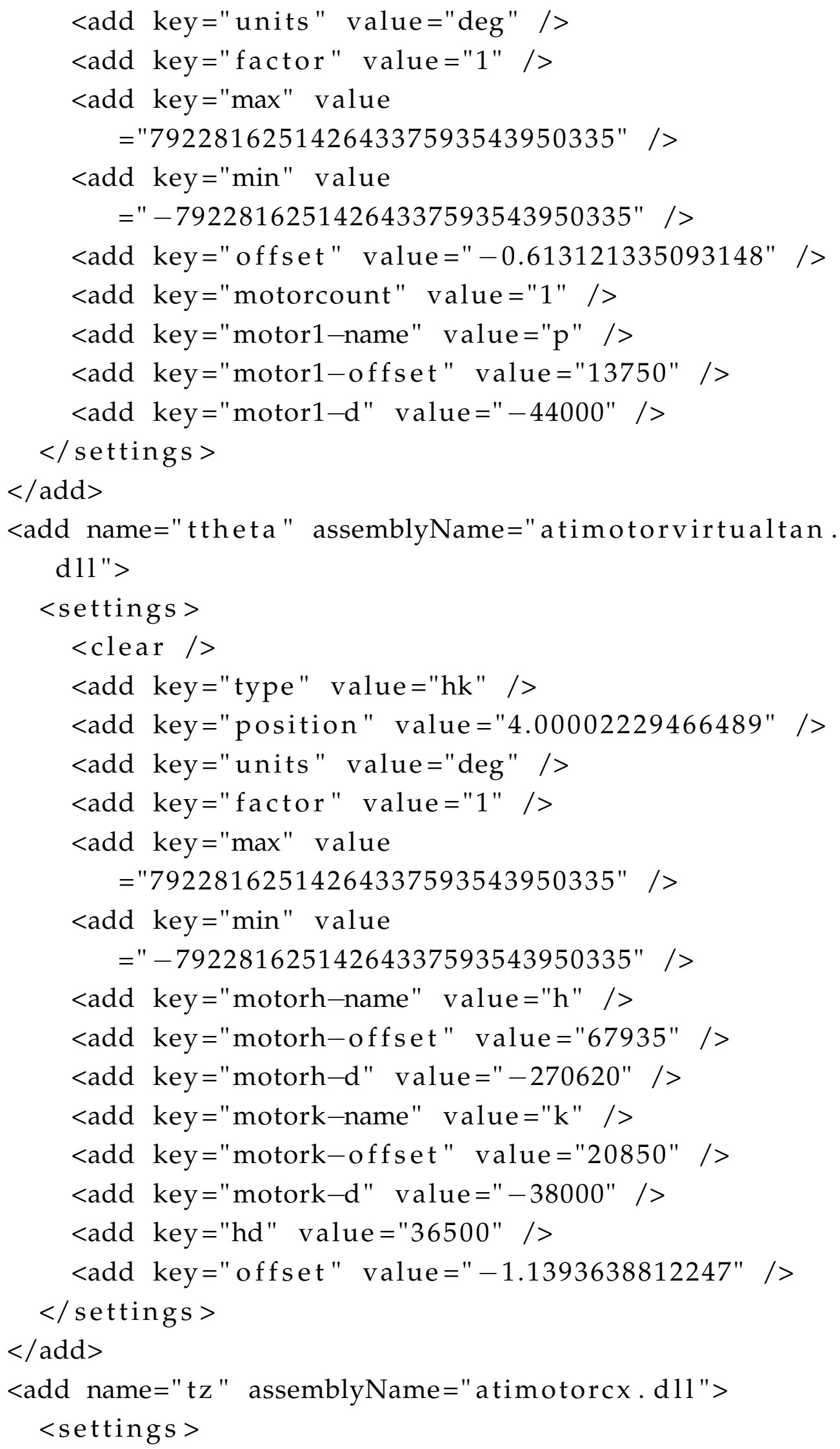




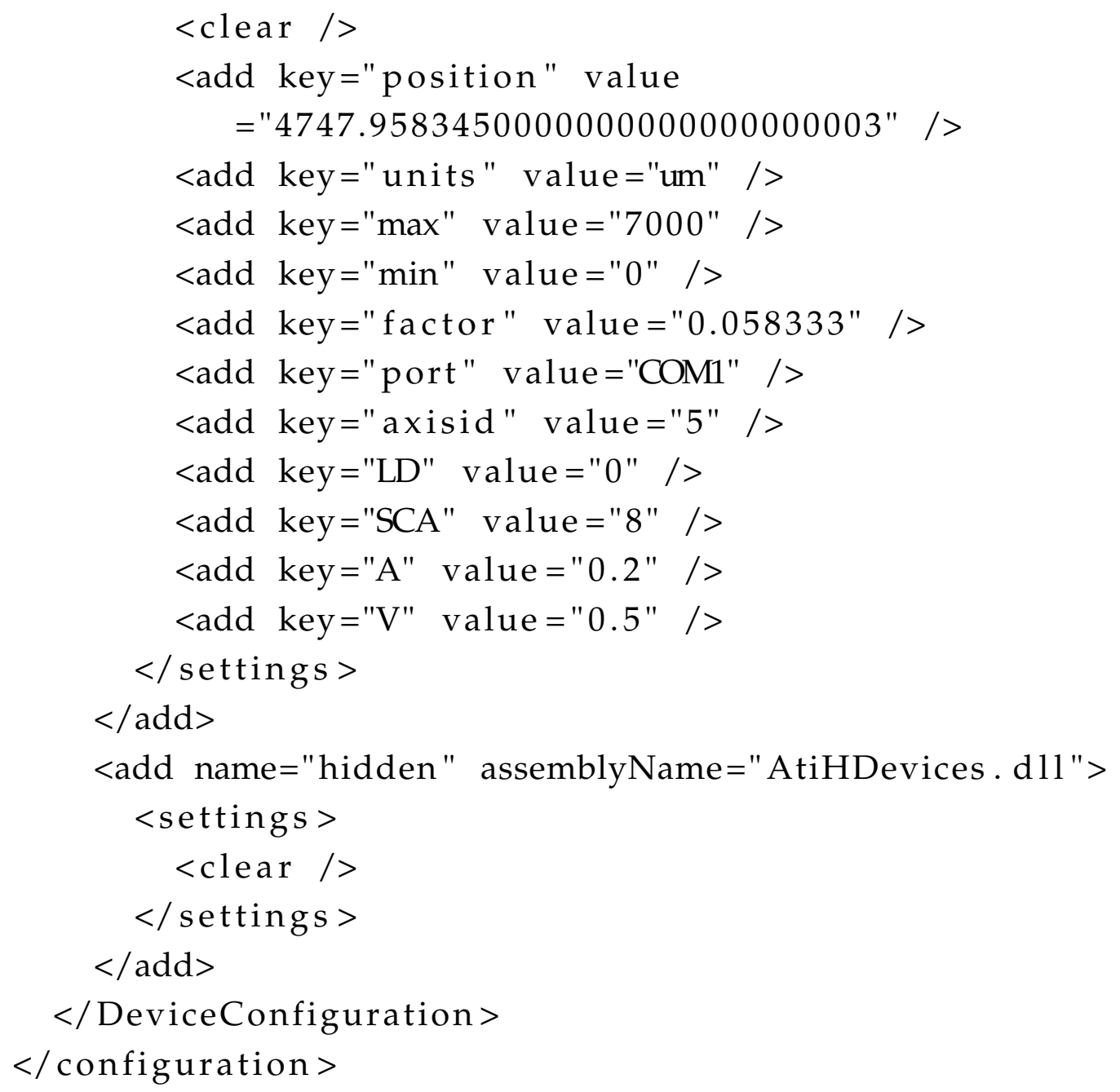

